\title{
Transcriptome Analysis of Ten Days Post Anthesis Elongating Fiber in the Upland Cotton (Gossypium hirsutum) Chromosome Substitution Line CS-B25
}

\author{
Chuan-Yu Hsu', Mark A. Arick II'1, Qing Miao,2,3, Sukumar Saha4, Johnie N. Jenkins4, \\ Mirzakamol S. Ayubov5, Ibrokhim Y. Abdurakhmonov5, Daniel G. Peterson', Din-Pow Ma ${ }^{2 *}$ \\ ${ }^{1}$ Institute for Genomics, Biocomputing \& Biotechnology, Mississippi State University, Mississippi State, MS, USA \\ ${ }^{2}$ Department of Biochemistry, Molecular Biology, Entomology and Plant Pathology, Mississippi State University, \\ Mississippi State, MS, USA \\ ${ }^{3}$ Department of Pharmacology, Weill Cornell Medical College, Cornell University, New York, NY, USA \\ ${ }^{4}$ USDA-ARS, Crop Science Research Laboratory, Mississippi State, MS, USA \\ ${ }^{5}$ Center of Genomics and Bioinformatics, Academy Sciences of Uzbekistan, Tashkent, Uzbekistan \\ Email: ^dma@bch.msstate.edu
}

How to cite this paper: Hsu, C.-Y., Arick II, M.A., Miao, Q., Saha, S., Jenkins, J.N., Ayubov, M.S., Abdurakhmonov, I.Y., Peterson, D.G. and Ma, D.-P. (2018) Transcriptome Analysis of Ten Days Post Anthesis Elongating Fiber in the Upland Cotton (Gossypium hirsutum) Chromosome Substitution Line CS-B25. American Journal of Plant Sciences, 9, 1334-1361. https://doi.org/10.4236/ajps.2018.96098

Received: March 27, 2018

Accepted: May 27, 2018

Published: May 30, 2018

Copyright $\odot 2018$ by authors and Scientific Research Publishing Inc. This work is licensed under the Creative Commons Attribution International License (CC BY 4.0).

http://creativecommons.org/licenses/by/4.0/

\section{(c) (i) Open Access}

\begin{abstract}
A chromosome substitution line, CS-B25, was developed by the substitution of chromosome pair 25 of Gossypium hirsutum TM-1 with the homologous pair of chromosome 25 from G. barbadense, a double haploid Pima 3-79 line. CS-B25 has improved fiber traits compared to its parent TM-1. To explore the molecule mechanisms underlying improved fiber traits, deep sequencing of total RNA was used to compare gene expression in fibers of CS-B25 and TM-1 at 10 days post anthesis (10-DPA). A total of 1872 differentially expressed genes (DEGs) were detected between the two lines, with 1175 up-regulated and 697 down-regulated in CS-B25. Gene Ontology (GO) enrichment analysis of the expression data by Generally Applicable Gene-set Enrichment (GAGE) and ReviGO indicated that the most prevalent Biological Process GO terms associated with DEGs included DNA-templated transcription, response to oxidative stress, and cellulose biosynthesis. Enriched Molecular Function GO terms included structural constituents of cytoskeleton, peroxidase activity, cellulose synthase (UDP-forming) activity, and transcription regulatory region sequence-specific DNA binding factors. GAGE was also used to find enriched KEGG pathways, and the highly represented pathways were Biosynthesis of Amino Acids, Starch and Sucrose Metabolism, Phenylpropanoid Biosynthesis, Protein Processing in Endoplasmic Reticulum, and Plant Hormone Signal Transduction. Many of the identified DEGs are involved in cy-
\end{abstract}


toskeleton and cell wall metabolism. The results of gene expression data have provided new insight into the molecular mechanisms of fiber development during the fiber elongation stage and would offer novel candidate genes that may be utilized in cotton fiber quality improvement.

\section{Keywords}

Chromosome Substitution Lines, Differentially Expressed Genes, Fiber

Development, Quantitative Real-Time PCR, RNA Sequencing, Upland Cotton

\section{Introduction}

Cotton fibers are seed trichomes and differentiated from the epidermal cells of a developing seed. The development of cotton fiber consists of four partially overlapping stages: initiation, elongation (primary wall synthesis), secondary wall thickening, and maturation [1]. Fiber cells initiate from 3 days before anthesis to 3 days post anthesis (DPA). After initiation, fiber cells immediately enter into the elongation stage. Fiber elongation reaches peaks at $6-12 \mathrm{DPA}$, but can last up to 22 - 26 DPA. The secondary wall synthesis begins at $16-19$ DPA, reaches a maximal rate at $25 \mathrm{DPA}$, and lasts up to $40 \mathrm{DPA}$. Fiber maturation, the last stage of fiber development, occurs at 40 - 50 DPA [1]. Fiber cell initiation and elongation are the two most important developmental stages with regard to fiber quality [2].

Cotton provides the largest renewable natural fiber for the textile industry and is also an important source of food, feed, fuel and other products [3]. Over $90 \%$ of the world cotton production is from Upland cotton, Gossypium hirsutum L. [4]. Upland cotton is widely adapted and has a high percentage of lint fiber and a relatively high yield. Pima cotton ( $G$. barbadense L.), the second most widely-grown cotton species, generally has a lower lint percentage and lower yield, but its fiber qualities are superior to Upland cotton in terms of length, strength, and fineness. The Pima fiber offers superior spinning and manufacturing performance and better textile products, and thus normally commands a $30 \%$ to $50 \%$ price advantage over fiber from high-yielding Upland cottons. Pima cotton, however, requires long growing seasons to produce profitable yields of high quality fiber. One of the major goals of cotton breeders is to develop Upland cotton with Pima-like elite fiber quality combined with high yield and adaptability. Combining the adaptability and yield of Upland cotton with the fiber quality of $G$. barbadense into an Upland cotton cultivar would maximize quality and productivity in an increasingly competitive market. However, crosses between $G$. hirsutum and G. barbadense at the whole genome level have not been highly successful due to technical and biological challenges associated with the conventional methods of interspecific introgression. An alternative approach to interspecific introgression is to use alien chromosome substitution lines where individual chromosomes or chromosome arms of $G$. barbadense are substituted into 
G. hirsutum. Seventeen interspecific chromosome substitution lines (CS-B lines) of Upland cotton (TM-1 background) containing whole chromosomes or chromosome arms of $G$. barbadense (Pima 3-79) chromosomes have been developed and released to the public [5]. These lines are genetically identical except that each differs by the replacement of a specific homologous pair of chromosomes from Pima 3-79 (G. barbadense) into the Upland background. CS-B25, a line in which the chromosome 25 pair of TM-1 has been replaced with the homologous chromosome 25 pair from $G$. barbadense, exhibits several improved fiber quality traits including increased fiber length, increased fiber strength, and lower micronaire [6] [7] [8] [9] [10]. Results from crosses of CS-B25 with selected USA improved cultivars also demonstrated that CS-B25 had several beneficial alleles with additive gene effects for improving fiber strength and decreasing micronaire in Upland cultivars [9] [10]. Additionally, CS-B25 has some beneficial alleles for improving agronomic traits with dominance genetic effects for lint percentage, seed cotton, and lint yield [11].

One of the serious impediments in cotton genetic improvement is the paucity of information of genes and their association with important fiber traits. The CS-B lines, being isogenic lines with the recurrent parent TM-1 except for the substituted chromosome pair from the alien species of $G$. barbadense, provide excellent analytical tools to dissect genetically complex fiber traits at the molecular level. The CS-B22sh line (chromosome 22 short arm substitution from Pima 3 - 79 into TM-1) was first used as a tool to identify differentially expressed transcripts in cotton [12]. The authors reported that 36 genes were differentially expressed in 15-DPA fiber in CS-B22sh, including genes that encoded tubulins, actin, cellulose synthase, glycosyl hydrolase, glycoside hydrolase, glycoside pyruvate decarboxylase and genes involved in signal transduction and nucleic acid, protein and lipid metabolism. Another report showed that CS-B22sh had positive additive effect on decreasing micronaire and a positive homozygous dominance effect on fiber strength [9].

In this study a genome wide transcriptome analysis was used to identify differentially expressed genes (DEGs) in 10-DPA fibers in CS-B25 as compared to TM-1. These DEGs and KEGG pathways analysis have provided insight into the mechanisms of fiber development during the fiber elongation stage and offered novel candidate genes that can be utilized in the genetic improvement of fiber quality.

\section{Materials and Methods}

\subsection{Plant Materials and Growth Conditions}

G. hirsutum L. cv. TM-1 and G. hirsutum CS-B25 plants were grown in the field at the R. R. Foil Plant Science Research Center at Mississippi State University (geographic coordinate: latitude N33.47 and longitude W88.77). TM-1 (Texas Marker-1) is a homozygous inbred line, serving as the genetic and cytogenetic standard for Upland cotton, G. hirsutum. TM-1 was developed by selection from 
Deltapine 14 at Texas A\&M AgriLife Research, College Station, Texas. CS-B25 line was developed using a special cytogenetically modified backcross-inbred breeding method [5]. The method used a specific hypoaneuploid type of G. hirsutum, primary monosomic for chromosome 25, as the recurrent parent which was previously backcrossed for more than 30 generations to inbred TM-1 and was thus quasi-isogenic hypoaneuploid type for chromosome 25 to TM-1. The recurrent hypoaneuploid for chromosome 25 monosomic plant was crossed with Pima 3-79, a double haploid G. barbadense line, as the male parent in developing the CS-B25 line. The cytologically identified monosomic F1 hybrid was used in a program with continuous backcrossing with TM-1 as the recurrent parent until the BC5F1 hypoaneuploid F1 plant was recovered. Upon identification, a hypoaneuploid BC5F1 segregate was selfed to identify a disomic, true-breeding disomic substitution. The substitution line was named as CS-B25 according to the expectedly substituted alien chromosome for 25 from the $G$. barbadense 3-79. The CS-B25 line is isogenic to the parent TM-1 for 25 chromosome pairs except the pair of chromosome 25 from the $G$. barbadense 3-79. Flowers were tagged on the day of anthesis (0 DPA). Cotton bolls at 10-DPA were harvested. Fibers were carefully dissected from cotton bolls, immediately frozen in liquid nitrogen, and then stored at $-80^{\circ} \mathrm{C}$ for RNA extraction.

\subsection{Extraction and Purification of Fiber RNA}

Total RNA was isolated from 10-DPA fibers of TM-1 and CS-B25 lines using a modified hot borate method [13]. Genomic DNA contamination in the RNA samples was removed by DNase I (Promega, Madison, Wisconsin, USA). After DNase treatment, other potential contaminants were removed from the RNA sample with a Qiagen RNasey Mini Kit (Qiagen, Valencia, California, USA). The quality of the total RNA sample was pre-checked on a $1 \%-1.2 \%(\mathrm{w} / \mathrm{v})$ agarose gel by electrophoresis, and the RNA concentration was estimated with a Nanodrop 1000 Spectrophotometer (NanoDrop Technologies, Wilmington, Delaware, USA) and a Qubit ${ }^{\oplus}$ Fluorometer by using the Qubit RNA BR Assay Kit (Invitrogen, Carlsbad, California, USA). The quality of the total RNA was finally determined using both agarose gel electrophoresis and a Bioanalyzer 2100 system (Agilent Technologies, Santa Clara, California, USA).

\subsection{RNA Library Preparation, Illumina Sequencing and Data Collection}

The isolated total RNA samples from 10-DPA fibers (1.5 $\mu \mathrm{g}$ from each sample) were used to construct strand-specific cDNA libraries using the TruSeq Stranded mRNA Sample Preparation Kit (Illumina, San Diego, California, USA) according to the manufacturer's instructions. Three biological replicate samples of the two cotton lines CS-B25 and TM-1 were used to generate 6 RNA-Seq libraries which were pooled and sequenced on one lane of an Illumina HiSeq 2000 sequencer (pair end sequencing, 50 bp per end) (Illumina, San Diego, California, 
USA). The raw reads were mapped to the G. hirsutum (AD1) NAU-NBI genome [14] using Tophat 2 [15]. Next HTSeq [16] was used to estimate gene expression from the Tophat alignments. The mRNA sequencing reads were deposited into NCBI Sequence Read Archive (SRA) with accession numbers SRR6015224-6 and SRR6015221-3 for TM-1 and CS-B25, respectively.

\subsection{Identification and Functional Analysis of Differentially Expressed Genes}

EdgeR [17], a Bioconductor (http://www.bioconductor.org/) software package for differential gene expression analysis, was used to identify DEGs in CS-B25 10-DPA fibers via comparison with TM-1 10-DPA fibers. Generalized linear models were used to test for differential gene expression between CS-B25 and TM-1. Genes with an FDR adjusted p-value $\leq 0.05$ were considered differentially expressed. GAGE [18], a Bioconductor software package for differential gene set analysis, was used to identify differentially expressed GO terms [19] and KEGG pathways [20]. GO gene sets were compiled from the functional annotation available from CottonGen [21]. REViGO [22] was used to summarize and visualize the enriched GO terms. Gene expression data was mapped to Arabidopsis KEGG pathways using gene homologies reported on CottonGen.

\subsection{Quantitative Real-Time PCR}

Twelve DEGs including 8 up-regulated and 4 down-regulated in CS-B25 were selected for validation using qRT-PCR. One microgram of fiber 10-DPA total RNA was used as template for first-strand cDNA synthesis using the random hexamer (Promega, Madison, Wisconsin, USA) and M-MLV reverse transcriptase (Promega, Madison, Wisconsin, USA) according to the manufacturer's instructions. The synthesized cDNA was then used as template for qRT-PCR reactions using PowerUp SYBR Green Master Mix and a QuantStudio 5 Real-Time PCR System (Applied Biosystems, Waltham, Massachusetts, USA). Cotton 18S rRNA was used as a reference gene to normalize the expression levels of RT-PCR products [23]. All reactions were performed with three replicates. The $2^{-\Delta \Delta C t}$ method $[\Delta \mathrm{Ct}=\mathrm{Ct}$ (differentially expressed gene) $-\mathrm{Ct}(18 \mathrm{~S}$ rRNA), $\Delta \Delta \mathrm{Ct}=\Delta \mathrm{Ct}$ $(\mathrm{CS}-\mathrm{B} 25)-\Delta \mathrm{Ct}(\mathrm{TM}-1), 2^{-\Delta \Delta \mathrm{Ct}}=$ Relative Expression] was used to calculate relative expression of differentially expressed genes [24]. All primers were designed by using Primer3 plus and the sequences of the primers are listed in Table S1.

\subsection{Analysis of SSR DNA Markers Associated with Chromosome 25}

Seven chromosome 25-specific nuclear SSR markers associated with improved fiber traits of fineness (micronaire), length, and strength were selected from the previous QTL mapping of fiber quality loci (see Table S2). These QTLs identify genetic relationship among the selected improved G. hirsutum and G. barbadense accessions including the recurrent parent TM-1 and the donor parent Pima 3-79 of the CS-B25 line. The seven chromosome-specific SSR markers were 
used to construct a dendrogram based on the average coefficient of similarity using JMP Genomics program (SAS $\left.{ }^{\mathrm{TM}}\right)$. The method of Egamberdiev et al. [25] was used to verify SSRs via PCR amplification and capillary electrophoresis.

\section{Results}

\subsection{Transcriptome Analysis of 10 DPA Fiber from CS-B25 and TM-1}

Approximately 300 million total sequence reads of $50 \mathrm{bp}$ were generated for this study. Analysis of sequencing data of CS-B25 and TM-1 from the six RNA libraries revealed that more than $80 \%$ of reads were mapped to the TM- 1 reference genome. Table 1 shows the total reads statistics of the six libraries. Of the 70,478 genes annotated in the $G$. hirsutum reference, 57,366 had at least one sequence read alignment. A total of 1870 genes (Table S3) were found to be differentially expressed in 10-DPA fiber when comparing CS-B25 to the TM-1 control at the false discovery rate (FDR) cutoff of $5 \%$ (adjusted $\mathrm{p} \leq 0.05$ ) and an absolute value of $\log _{2}$ ratio $\geq 1$. Among them, 1173 and 697 genes were up-regulated and down-regulated in CS-B25, respectively.

\subsection{Gene Function Annotation by GO Enrichment Analysis}

GO enrichment analysis by GAGE and ReviGO indicated that Biological Process GO terms related to DNA-templated transcription, response to oxidative stress, and cellulose biosynthetic processes were enriched (Figure 1(a)). In addition, the Molecular Function GO terms including structural constituent of cytoskeleton, peroxidase activity, cellulose synthase (UDP-forming) activity, and transcription regulatory region sequence-specific DNA binding were predominant (Figure 2(a)). The 57,366 G. hirsutum genes with expression data mapped to 14,610 homologous Arabidopsis genes were used to find enriched KEGG pathways. Most enriched pathways were found to be related to biosynthesis of secondary metabolites, biosynthesis of amino acids, starch and sucrose metabolism, amino sugar and nucleotide sugar metabolism, and plant hormone signal transduction (Table 2). The genes differentially expressed in CS-B25 10-DPA fibers were functionally annotated, and representatives of these differentially expressed genes (DEGs) are listed in Table 3 and Table S3.

Table 1. Total reads statistics of RNA-Seq data

\begin{tabular}{ccccccc}
\hline $\begin{array}{c}\text { RNA-Seq } \\
\text { library ID }\end{array}$ & TM1-1 & TM1-2 & TM1-3 & CS-B25-1 & CS-B25-2 & CS-B25-3 \\
\hline $\begin{array}{c}\text { Total reads } \\
\text { Total }\end{array}$ & $48,362,375$ & $44,800,820$ & $106,254,636$ & $46,940,992$ & $45,866,594$ & $48,500,739$ \\
$\begin{array}{c}\text { unique reads } \\
\text { Overall \% }\end{array}$ & $14,097,884$ & $12,148,341$ & $16,029,773$ & $16,444,626$ & $15,765,544$ & $16,855,253$ \\
Concordant & 99.2 & 99.2 & 99.2 & 99.3 & 99.3 & 99.1 \\
\hline
\end{tabular}




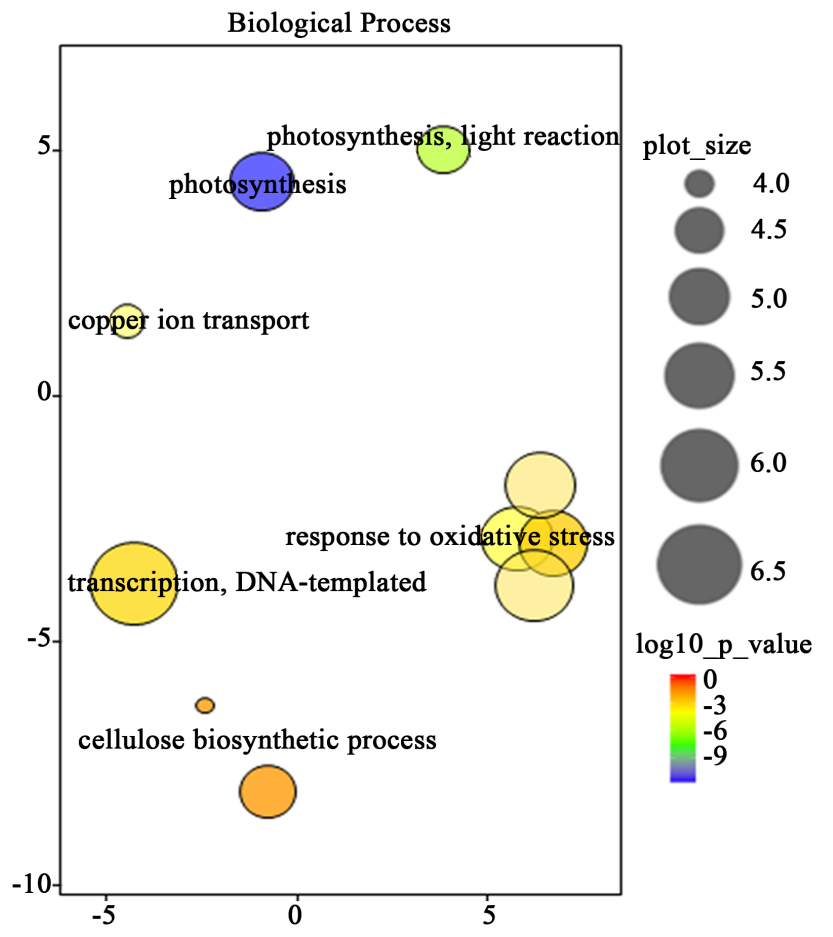

(a)

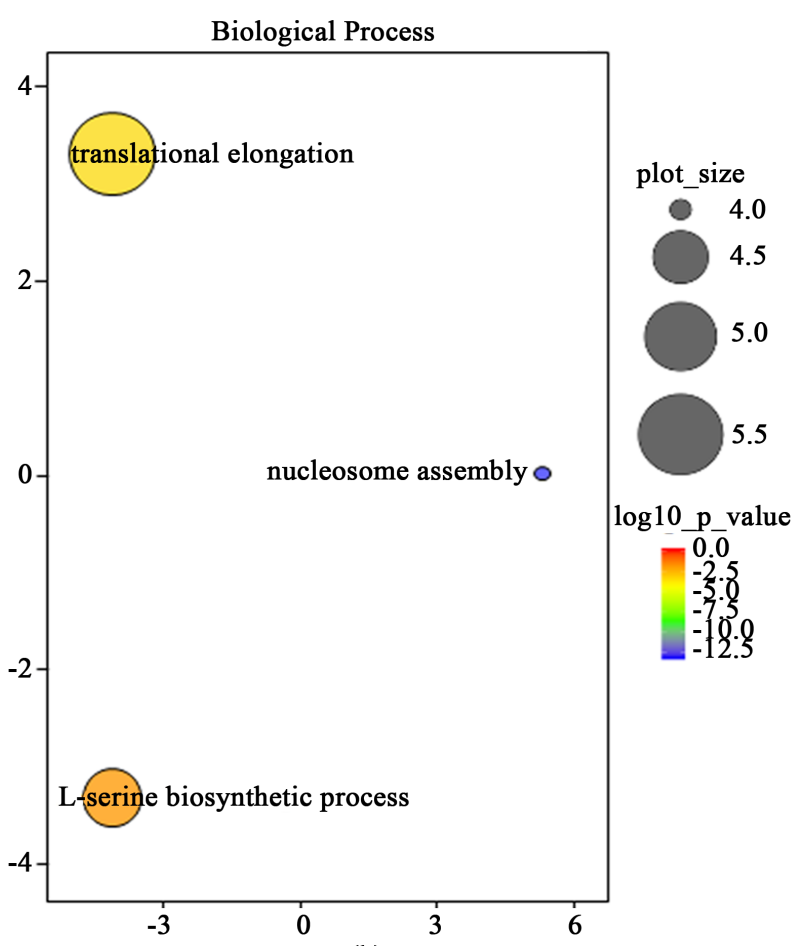

(b)

Figure 1. A ReViGo scatter plot of GO analyses in biological process from (a) up-regulated and (b) down-regulated DEGs in the CS-B25 10-DPA fibers. Related GO terms are clustered and presented with bubbles with similar color shades. Bubble colors represent $\mathrm{p}$-values and bubble sizes indicate the relative frequency of the GO terms.
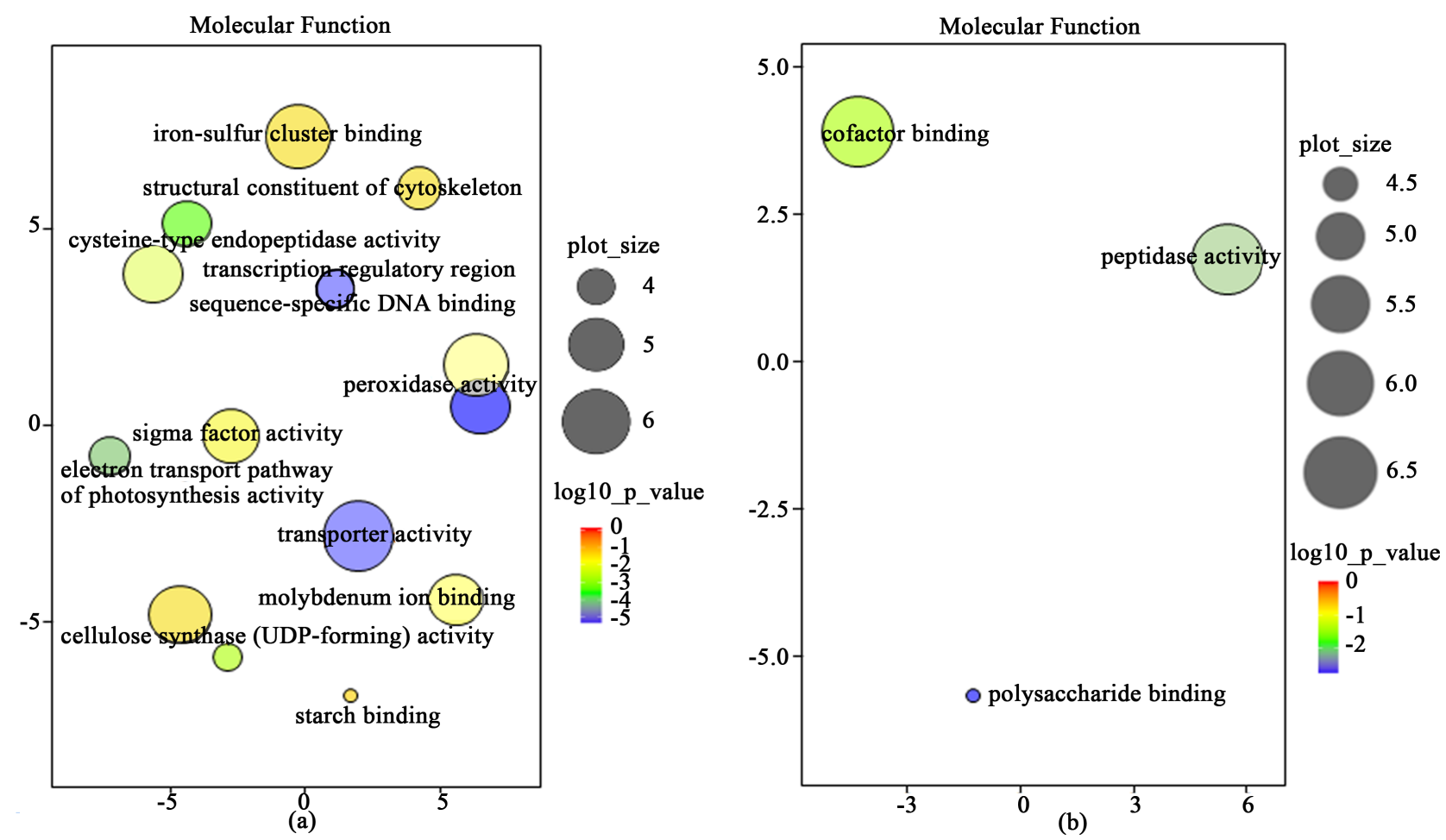

Figure 2. A ReViGo scatter plot of GO analyses in molecular function from (a) up-regulated and (b) down-regulated DEGs in the CS-B25 10-DPA fibers. Related GO terms are clustered and presented with bubbles with similar color shades. Bubble colors represent $\mathrm{p}$-values and bubble sizes indicate the relative frequency of the GO terms. 
Table 2. KEGG pathway analysis of KOs.

\begin{tabular}{|c|c|c|}
\hline Pathway & KOs & Pathway ID \\
\hline Metabolic pathways & 113 & ko01100 \\
\hline Biosynthesis of secondary metabolites & 71 & ko01110 \\
\hline Biosynthesis of antibiotics & 27 & ko01130 \\
\hline Microbial metabolism in diverse environments & 19 & ko01120 \\
\hline Biosynthesis of amino acids & 17 & ko01230 \\
\hline Ribosome & 15 & ko03010 \\
\hline Starch and sucrose metabolism & 14 & ko00500 \\
\hline Plant hormone signal transduction & 13 & ko04075 \\
\hline Amino sugar and nucleotide sugar metabolism & 11 & ko00520 \\
\hline Carbon metabolism & 11 & ko01200 \\
\hline Plant-pathogen interaction & 10 & ko04626 \\
\hline Photosynthesis & 10 & ko00195 \\
\hline
\end{tabular}

Table 3. Annotation of genes differentially expressed in CS-B25 10-DPA fibers.

\begin{tabular}{|c|c|c|c|}
\hline Gene ID & Tair 10 ID & Description & $\mathrm{FC}^{*}(\mathrm{CS}-\mathrm{B} 25 / \mathrm{TM}-1)$ \\
\hline \multicolumn{4}{|c|}{ Hormone signal transduction } \\
\hline Gh_A08G1512 & AT4G16770 & 1-aminocyclopropane-1-carboxylate oxidase-like & 8.59 \\
\hline Gh_A06G1558 & AT5G04530 & 3-ketoacyl-CoA synthase 19 & 0.045 \\
\hline Gh_D10G2187 & AT5G04530 & 3-ketoacyl-CoA synthase 21-like & 3.21 \\
\hline GH_A04G0224 & AT1G52820 & gibberellin 20 oxidase & 11 \\
\hline Gh_A04G0228 & AT1G52820 & gibberellin 3-beta-dioxygenase 4 & 10.4 \\
\hline Gh_A06G0045 & AT2G18420 & gibberellin-regulated family protein & 4.97 \\
\hline Gh_A01G2049 & AT1G78440 & gibberellin 2-oxidase 1 & 0.058 \\
\hline Gh_D08G2151 & AT3G51770 & ethylene-overproduction 1-like TRR-containing protein & 17.77 \\
\hline Gh_A07G0377 & AT5G07580 & Ethylene-responsive transcription factor ERF106 & 8.76 \\
\hline Gh_A04G0096 & AT5G55340 & long-chain alcohol O-fatty-acyltransferase 5 & 277.51 \\
\hline Gh_A04G0106 & AT1G50640 & ethylene responsive element binding factor 3 & 0.008 \\
\hline Gh_D06G0023 & AT1G75540 & zinc finger transcription factor Constans & 23.45 \\
\hline Gh_A08G0537 & AT2G44840 & ethylene responsive 13 & 0.055 \\
\hline Gh_A08G0780 & AT5G57740 & RING E3 ligase XBAT32 & 0.0009 \\
\hline Gh_A02G0011 & AT5G01990 & auxin influx carrier family protein & 215.15 \\
\hline Gh_A03G1429 & AT5G54510 & auxin-responsive GH3 family protein & 73.05 \\
\hline Gh_D02G2389 & AT4G14550 & auxin-responsive protein IAA14-like & 40.88 \\
\hline Gh_A06G0351 & AT1G77690 & auxin influx transport protein & 7.41 \\
\hline
\end{tabular}




\section{Continued}

\begin{tabular}{|c|c|c|c|}
\hline Gh_D02G2219 & AT4G34770 & SAUR-like auxin-responsive protein & 0.047 \\
\hline Gh_D06G2010 & AT3G15540 & auxin-induced protein AUX22-like & 0.1 \\
\hline Gh_A03G1429 & AT5G54510 & indole-3-acetic acid-amido synthetase GH3.6-like & 73.05 \\
\hline Gh_A10G2215 & AT5G60850 & zinc finger transcription factor DOF5 & 74.64 \\
\hline Gh_A13G0392 & AT2G14960 & auxin-responsive $\mathrm{GH} 3$ family protein & 0.12 \\
\hline Gh_D09G0013 & AT2G34740 & protein phosphatase $2 \mathrm{C}$ family protein & 70.17 \\
\hline Gh_A02G0999 & AT5G59220 & highly ABA-induced PP2C gene 1 & 0.096 \\
\hline \multicolumn{4}{|c|}{ Cell wall biosynthesis/modification } \\
\hline Gh_A08G0457 & AT2G04780 & fasciclin-like arabinogalactan protein 7 & 3338.37 \\
\hline Gh_D08G0544 & AT2G04780 & fasciclin-like arabinogalactan protein 7 & 210.99 \\
\hline Gh_D10G2231 & AT1G35710 & LRR receptor-like serine/threonine protein kinase & 293.42 \\
\hline Gh_D12G0597 & AT5G44700 & LRR receptor-like serine/threonine protein kinase & 21.09 \\
\hline Gh_D02G0280 & AT5G46330 & LRR receptor-like serine/threonine protein kinase & 23.75 \\
\hline Gh_D08G0544 & AT2G04780 & Ascorbate peroxidase 2 & 210.99 \\
\hline Gh_A04G0652 & AT3G09640 & Ascorbate peroxidase 2 & 49.27 \\
\hline Gh_A09G1963 & AT4G23990 & cellilose synthase-like protein G3 isoform X1 & 117.67 \\
\hline Gh_D09G2166 & AT4G23990 & cellulose synthase-like protein G3 & 155.29 \\
\hline Gh_D02G0079 & AT2G43820 & UDP-glycosyltransferase 74-F2-like & 120.09 \\
\hline Gh_D13G0415 & AT4G34131 & UDP-glucosyltransferase 73B3 & 0.0037 \\
\hline Gh_Sca004909G03 & AT2G43820 & UDP-glucosyltransferase 74F2 & 0.084 \\
\hline Gh_D11G0846 & AT2G45220 & pectin methylesterase 2 & 183.15 \\
\hline Gh_A11G0727 & AT2G45220 & pectin methylesterase 2 & 208.35 \\
\hline Gh_A08G0741 & AT5G56350 & pyruvate kinase, cytosolic isozyme & 369.33 \\
\hline Gh_D02G0381 & AT2G48130 & non-specific lipid transfer protein-like protein & 232.45 \\
\hline Gh_A02G0317 & AT3G22600 & lipid transfer protein-like protein & 219.08 \\
\hline Gh_A01G1193 & AT2G37870 & non-specific lipid transfer protein-like & 45.76 \\
\hline Gh_D07G2095 & AT2G03090 & expansin A15 & 0.039 \\
\hline Gh_Sca012883G01 & AT5G23860 & tubulin beta- 8 & 30.51 \\
\hline Gh_D09G1351 & AT2G36710 & pectin lyase-like superfamily protein & 155.96 \\
\hline Gh_D08G1862 & AT3G14300 & pectinesterase family protein & 87.69 \\
\hline Gh_A09G1349 & AT2G36710 & pectin lyase-like superfamily protein & 25.6 \\
\hline Gh_A07G1458 & AT3G07010 & pectin lyase-like superfamily protein & 0.061 \\
\hline Gh_D08G2205 & AT3G05610 & pectin methylesterase inhibitor superfamily & 0.017 \\
\hline Gh_A06G0439 & AT3G12500 & chitinase & 13.73 \\
\hline
\end{tabular}




\section{Continued}

\begin{tabular}{|c|c|c|c|}
\hline & & & \\
\hline Gh_A12G2714 & AT5G24090 & chitinase A & 0.092 \\
\hline Gh_D12G2669 & AT5G24090 & chitinase A & 0.033 \\
\hline & & nal regulation of cellular metabolic process & \\
\hline Gh_A08G0993 & AT1G63910 & myb domain protein 103 & 172.34 \\
\hline Gh_A13G1873 & AT5G12870 & transcription factor MYB46 & 155.87 \\
\hline Gh_D11G2061 & AT3G49690 & myb domain potein 84 & 83.18 \\
\hline Gh_D13G1452 & AT2G32460 & myb domain protein 101 & 45.57 \\
\hline Gh_D08G1266 & AT1G63910 & myb domain protein 103 & 143.47 \\
\hline Gh_A09G2017 & AT3G24310 & myb-related protein 305 -like & 40.58 \\
\hline Gh_D11G1132 & AT2G47190 & myb domain protein 2 & 40.54 \\
\hline Gh_D05G1913 & AT3G18100 & myb domain protein $4 \mathrm{r} 1$ & 20.74 \\
\hline Gh_A02G1357 & AT5G16770 & myb domain protein 9 & 0.067 \\
\hline Gh_D05G2967 & AT1G14600 & myb family transcription factor & 30.75 \\
\hline Gh_A08G0907 & AT2G46680 & homeobox protein 7 & 97.47 \\
\hline Gh_D02G1259 & AT5G39760 & homeobox protein 23 & 58.09 \\
\hline Gh_A04G0732 & AT5G02030 & POX (plant homeobox) family protein & 31.45 \\
\hline Gh_A08G0909 & AT3G61890 & homeobox protein 12 & 13.57 \\
\hline Gh_A03G0158 & AT5G41410 & POX (plant homeobox) family protein & 9.73 \\
\hline Gh_D06G1066 & AT5G25200 & KNOTTED1-like homeobox gene 3 & 10.89 \\
\hline Gh_A11G2219 & AT1G14440 & homeobox protein 31 & 10.13 \\
\hline Gh_D05G1962 & AT1G46480 & WUSCHEL related homeobox 4 & 28.28 \\
\hline Gh_A05G2722 & AT1G23380 & KNOTTED1-like homeobox gene 6 & 0.065 \\
\hline Gh_A03G0887 & AT1G69490 & NAC transcription factor 29 & 60.7 \\
\hline Gh_A08G1691 & AT4G28530 & NAC domain containing protein 74 & 50.5 \\
\hline Gh_A05G1339 & AT4G28500 & NAC domain containing protein 73 & 9.99 \\
\hline Gh_A08G1086 & AT4G01550 & NAC domain containing protein 69 & 0.0032 \\
\hline Gh_A11G1172 & AT2G30590 & WRKY transcription factor 21 & 16.78 \\
\hline Gh_A02G1042 & AT4G23550 & WRKY transcription factor 29 & 3.27 \\
\hline & & Phenylpropanoid biosynthesis & \\
\hline Gh_A02G1258 & AT2G02400 & cinnamoyl-CoA reductase 1 & 4.94 \\
\hline Gh_D01G1091 & AT4G24510 & shikimate O-hydroxycinnamoyltransferase & 33.16 \\
\hline Gh_A01G1688 & AT1G68540 & cinnamoyl-CoA reductase 2 & 49.79 \\
\hline Gh_D11G1234 & AT3G06350 & dehydroquinate dehydratase/shikimate dehydrogenase & 5.78 \\
\hline
\end{tabular}




\subsection{DEGs Involved in Hormone Synthesis and Signaling}

Three phytohormones of gibberellin, ethylene, and auxin have been shown to play important roles in cotton fiber development [26]. Several genes participating in gibberellin synthesis and in response to gibberellin stimulus were up-regulated in CS-B25 10-DPA fiber (Table 3 and Table S3). These included genes for gibberellin 20 oxidase (Gh_A04G0224, 11 fold upregulation), gibberellin 3-beta-dioxygenase 4 (Gh_A04G0228, 10.41 fold upregulation) and a gibberellin-regulated family protein (Gh_A06G0045, 4.97 fold upregulation). Genes involved in ethylene synthesis and signaling were also found to be up-regulated. These include genes for 1-aminocyclopropane-1-carboxylate oxidase (ACCO) (Gh_A08G1512, 8.59 fold and Gh_D05G1663, 4.91 fold), a key enzyme involved in ethylene biosynthesis [27] [28], ethylene-overproduction 1-like tetratricopeptide repeat (TRR)-containing protein (Gh_D08G2151, 17.77 fold upregulation) which is involved in chromatin remodeling and transcription regulation, ethylene responsive transcription factor ERF106 (Gh_A07G0377, up-regulated 8.76 fold), and a membrane bound long-chain-alcohol O-fatty-acyltransferase 5 (Gh_A04G0096, up-regulated 277.5 fold) involved in synthesis of very long-chain-fatty acids which had been shown to promote fiber elongation by stimulating an increase in ACCO transcript and ethylene production [28] (Table 3 and Table S3). In addition, genes involved in auxin synthesis and transport including auxin-responsible protein IAA14 (Gh_D02G2389, 40.9 fold), auxin transporter-like protein 3 (Gh_A06G0351, 7.41 fold), and auxin-responsive protein IAA14-like (Gh_A02G0341, 3.87 fold), auxin influx carrier family protein (Gh_A02G0011, 215 fold), and indole-3-acetic acid-amido synthetase GH3.6-like (auxin-responsive GH3 family protein) (Gh_A03G1429, 73.05 fold) were up-regulated. Genes encoding zinc finger transcription factors including CONSTANS (Gh_D06G0023, 23.44 fold, involved in ethylene biosynthesis) and DOF5 (Gh_A10G2215, 74.6 fold, affecting auxin homeostasis) [29] were also up-regulated. RING E3 ligase XBAT32 (Gh_A08G0780) was down-regulated 1,113 fold in CS-B25 compared to TM-1 (Table 3 and Table S3). XBAT32 reduces ethylene biosynthesis by decreasing the abundance of aminocyclopropane-1-carboxylic acid synthases [30], the enzymes participating in the first step of ethylene biosynthesis [31]. The gene Gh_D09G0013 encoding a protein phosphatase $2 \mathrm{C}$ family protein, a negative regulator of abscisic acid signaling [32], was up-regulated by 70.17 fold, suggesting that down regulation of ABA might contribute to a longer period of fiber elongation [33] and resulting in longer fibers in CS-B25.

\subsection{DEGs Involved in Cell Wall Synthesis}

Many genes involved in primary cell wall (PCW) biosynthesis were up-regulated in CS-B25 10-DPA fiber cells (Table 3 and Table S3). These include genes coding for fasciclin-like arabinogalactan proteins 7 (Gh_A08G0457, 3338.37 fold and Gh_D08G0544, 211 fold) [34], non-specific lipid transfer proteins (LTPs) 
(Gh_A02G0317, 219.1 fold, similar to At3G22600; Gh_D02G0381, 232.44 fold, similar to At2G48130 protein; Gh_A01G1193, 45.76 fold; Gh_A08G0129, 18.73 fold) [35], cellulose synthase-like protein G3 isoform X1 (Gh_A09G1963, 117.67 fold and Gh_D09G2166, 155.29 fold), UDP-glycosyltransferase 74F2-like (Gh_D02G0079, 120.09 fold), pectin methylesterase 2 (PME) (Gh_A11G0727, 208.35 fold and Gh_D11G0846, 183.15 fold) and pectin methylesterase 15 (Gh_D09G1351, 155.96 fold and Gh_A09G1349, 25.63 fold) [36]. Other genes related to the cytoskeleton, including tubulins beta 8 (Gh_Sca012883G01) was also up-regulated 30.5 fold. It had been suggested that specific tubulins might affect microtubule organization and microfibril deposition in elongating fiber cells [37]. An ABIL-1 gene encoding a subunit of the WAVE complex was highly expressed (1,262 fold upregulation). The WAVE complex is involved in regulation of actin and microtubule organization [38]. Genes for LRR receptor-like serine/threonine-protein kinases (Gh_D10G2231, 294 fold; Gh_D12G0597, 21.11 fold; Gh_D02G0280, 23.75 fold) were highly or moderately up-regulated, and they are known to participate in signal transduction pathways [39], cellulose deposition [40], and maintenance of cell wall integrity [41].

\subsection{Transcription Factors Involved in Fiber Development}

Many MYB-type transcription factors, found to regulate plant cell wall biosynthesis [42] [43], were also up-regulated in CS-B25 (Table 3 and Table S3). Genes for MYB transcription factors including MYB domain protein 103 (Gh_A08G0993, 172.34 fold; Gh_D08G1266, 143.47 fold; and Gh_A03G0166, 122.3 fold), MYB46 (Gh_D13G2261, 103.67 fold; Gh_A13G1873, 155.86 fold; Gh_D09G1082, 9.76 fold), MYB domain protein 305 (Gh_A09G2017, 40.58 fold) [44], MYB domain protein 101 (Gh_D13G1452, 45.57 fold), MYB domain protein 2 (Gh_D11G1132, 40.54 fold), MYB family transcription factor (Gh_D05G2967, 30.75), MYB44 (myb domain protein 4r1) (Gh_D05G1913, 20.74 fold), and MYB domain protein 84 (Gh_D11G2061, 83.18 fold) were up-regulated. Several homeobox leucine zipper proteins, including homeobox protein 7 (Gh_A08G0907, 97.47 fold), homeobox protein 23 (Gh_D02G1259, 58.08 fold), homeobox protein 12 (Gh_A08G0909, 13.57 fold) were up-regulated in CS-B25 as well. NAC transcription factors that activate MYBs, including NAC transcription factor 98 (Gh_D13G1561, 20.82 fold), NAC domain-containing protein 21/22-like (Gh_A08G1691, 50.56 fold), NAC transcription factor 100 (Gh_A11G0633, 7.36 fold), NAC-domain containing protein 73-like

(Gh_A05G1339, 9.98 fold), and NAC transcription factor 29-like (Gh_A03G0887, 60.55 fold), a negative regulator of $\mathrm{ABA}$ signaling with function in transition from active cell division to cell expansion, were also up-regulated. NAC domain-containing protein 67-like (Gh_A08G1086, 0.0032), however, was down-regulated. NAC transcription factors and NAC domain-containing proteins are a very large family in plants [45], and the SND protein classified as NAC domain protein 1 was reported in regulating secondary wall synthesis by targeting the MYB46 transcription factor [42]. WRKY transcription factors 21 
(Gh_A11G1172, 16.78 fold) and 29 (Gh_A02G1042, 3.27 fold) were also up-regulated in CS-B25.

\subsection{DEGs Involved in Phenylpropanoid Synthesis}

DEGs involved in phenylpropanoid biosynthesis were slightly up-regulated in CS-B25 (Table 3 and Table S3). Genes for cinnamoyl-CoA reductase 2 (Gh_A02G1258, 4.96 fold), caffeic acid 3-O-methyltransferase (Gh_A12G2227, 8.54 fold and Gh_A08G0929, 3.86 fold) (lignin synthesis), and bifunctional 3-dihydroquinate dehydratase/shikimate dehydrogenase (chloroplast-like isoform X1) (Gh_D11G1234, 5.78 fold) (lignin synthesis) were up-regulated. These expression data indicated that lignin/secondary cell wall synthesis also occurred in the fiber elongation step but the difference between CS-B25 and TM-1 was less dramatic.

\subsection{DEGs with Function in Oxidoreductase Activity}

Genes involved in oxidoreductase activity were up-regulated in CS-B25 10-DPA fiber (Table S3), which include genes for alcohol dehydrogenases (Gh_A04G1326, 878.37 fold), cytochrome P450 CYP736A12-like (Gh_A09G1848, 82.98 fold), cytochrome P450 84-A1 like (Gh_A11G3018, 58.28 fold), and cytochrome P450 72A1 (Gh_D02G0287, 25.78 fold). Genes for cytosolic pyruvate kinase (Gh_A08G0741, 369.33 fold) [46] and ascorbate peroxidase

(Gh_A04G0652, 49.27 fold) [47] involved in fiber elongation were also highly up-regulated in CS-B25 (Table S3).

In comparison to the numbers of up-regulated genes, fewer genes were down-regulated in CS-B25 10-DPA fiber (Table S3). Genes involved in translational elongation, L-serine biosynthetic processes, and nucleosome assembly in the Biological Process GO group are down-regulated (Figure 1(b)). Genes in Molecular Function including cofactor binding, polysaccharide binding, and peptidase activity are down-regulated (Figure 2(b)). Genes for ribosomal proteins including the 60S ribosomal protein L11 (Gh_D10G0336, 0.0696 fold), 60S ribosomal protein L5-like (Gh_D02G0103, 0.032 fold), 40S ribosomal protein S5-like (Gh_A04G0473, 0.0083 fold), and ribosomal protein L46 (Gh_A08G1142, 0.1118 fold) were down-regulated. Cyclin-J18 isoform X2 (Gh_A12G1420, 0.0145 fold), heme-binding-like protein (Gh_A04G0508, 0.0093 fold), 14-3-3-like protein (Gh_A01G0110, 0.027 fold), RAD-like transcription factor (Gh_A13G0293, 0.0042 fold), hypothetical protein (Gh_A10G1995, 0.0013 fold), Peptidase M20/M25/M40 family protein (Gh_A04G1433, 0.00235 fold), B3-domain-containing protein Os01g0234100-like (Gh_A01G0064, 0.0068 fold), E3 ubiquitin-protein ligase XBAT32 (E3-Ring finger, Gh_A08G0780, 0.000895 fold), and ethylene-responsive transcription factor 3-like (ERF3, Gh_A04G0106, 0.0081 fold) were down-regulated.

\subsection{Validation of Differentially Expressed Genes by qRT-PCR}

To test whether the gene expression results from RNA-seq were reliable, RNA 
transcripts of 16 DEGs detected in CS-B25 were selected for qRT-PCR analysis. All the 16 transcripts were successfully detected by qRT-PCR and 12 transcripts showed agreement with RNA-seq results (see Figure 3 ). Both RNA-seq and qRT-PCR detected that the expression levels of genes for lipid transfer protein and cellulose synthase were comparable, and CS-B25 had the two RNA transcripts that were more than 70-fold higher than those in TM-1. Based on the qRT-PCR data, the four genes encoding B3 domain-containing protein

Os01g0234100-like, E3 ubiquitin-protein ligase XBAT32, ethylene-responsive transcription factor 3-like, and 40S ribosomal protein S5-like had lower transcript levels in CS-B25, with fold change from 0.1 to 0.24 when compared with those in TM-1. The RNA-seq data, however, showed that TM-1 had higher transcripts levels of the four genes.

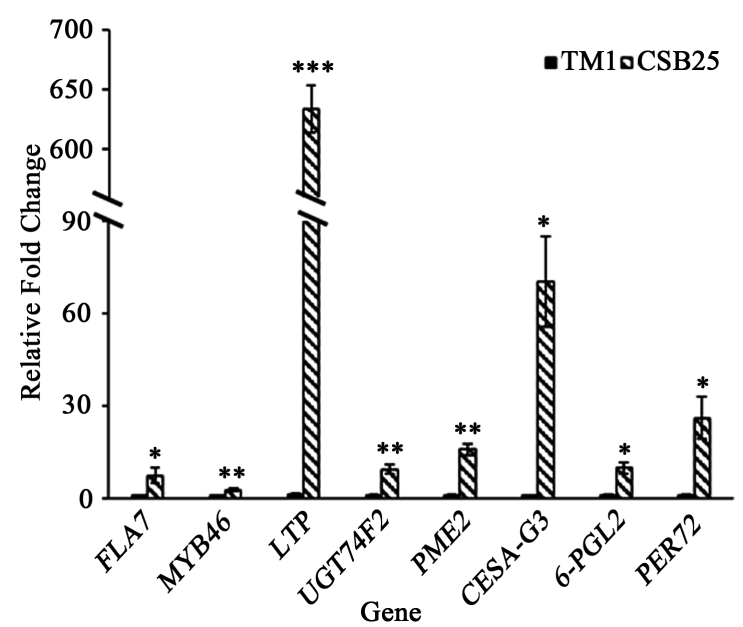

(a)

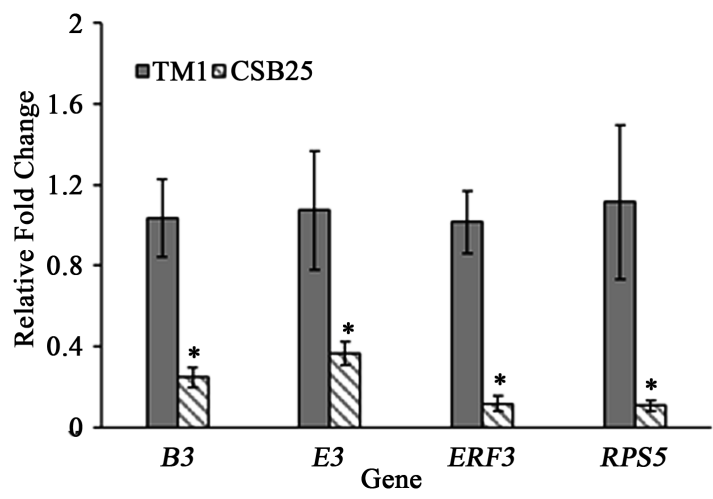

(b)

Figure 3. Quantitative RT-PCR analysis of differentially expressed genes in 10 DPA cotton fibers from CS-B25 and TM1 lines. Compared to TM1, eight selected up-regulated genes (a) and four selected down-regulated genes (b) identified in CS-B25 line from RNA-Seq analysis were validated with quantitative RT-PCR assays. At least three biological replicates were used in the assays, cotton $18 \mathrm{~S}$ rRNA was used as a reference gene to normalize the expression level. The significant difference in the expression of each transcript between CS-B25 and TM1 was analyzed by t-test ${ }^{*} \mathrm{p} \leq 0.05 ;{ }^{* *} \mathrm{p} \leq 0.005$; and ${ }^{* *} \mathrm{p} \leq$ 0.005). The error bars represent standard error of the mean. 


\subsection{Dendrogram Based on the Chromosome 25-Specific Simple Sequence Repeat (SSR) Markers}

Results from the dendrogram analysis divided all of the accessions into two broad groups of $G$. hirsutum and $G$. barbadense based on the average coefficient of similarity (IBD value indicating identical alleles), suggesting that the chromosome 25 is distinctly different at the molecular level and fiber traits between the two species accessions including the recurrent parent TM-1 and the donor parent Pima 3-79 of the CS-B25 line (Figure 4). Pima 3-79, the donor parent, was clustered with other improved $G$. barbadense accessions in the dendrogram based on the chromosome 25-specific nuclear SSR markers associated with improved fiber traits from previous reports. This observation suggests that the substituted alien species chromosome 25 pair in CS-B25 would have the potential to carry genes of improved fiber traits including those associated with micronaire, fiber length and fiber strength. The results from field experiments demonstrated that the substituted chromosome 25 pair from $G$. barbadense in CS-B25 had significant effects on improved micronaire (Figure 5), which is one of important fiber quality traits used in determining fiber price.

\section{Discussion}

Results from GO enrichment analysis by GAGE and ReviGO indicated that many genes were up-regulated in CS-B25 10-DPA fiber with the highest fold change (FC) in expression of the gene coding for a fasciclin-like arabinogalactan protein 7 (AGP, Gh_A08G0457, 3338.37 fold upregulation) in CS-B25 compared to TM-1 (Table 3 and Table S3). Using over-expression and RNAi approaches,

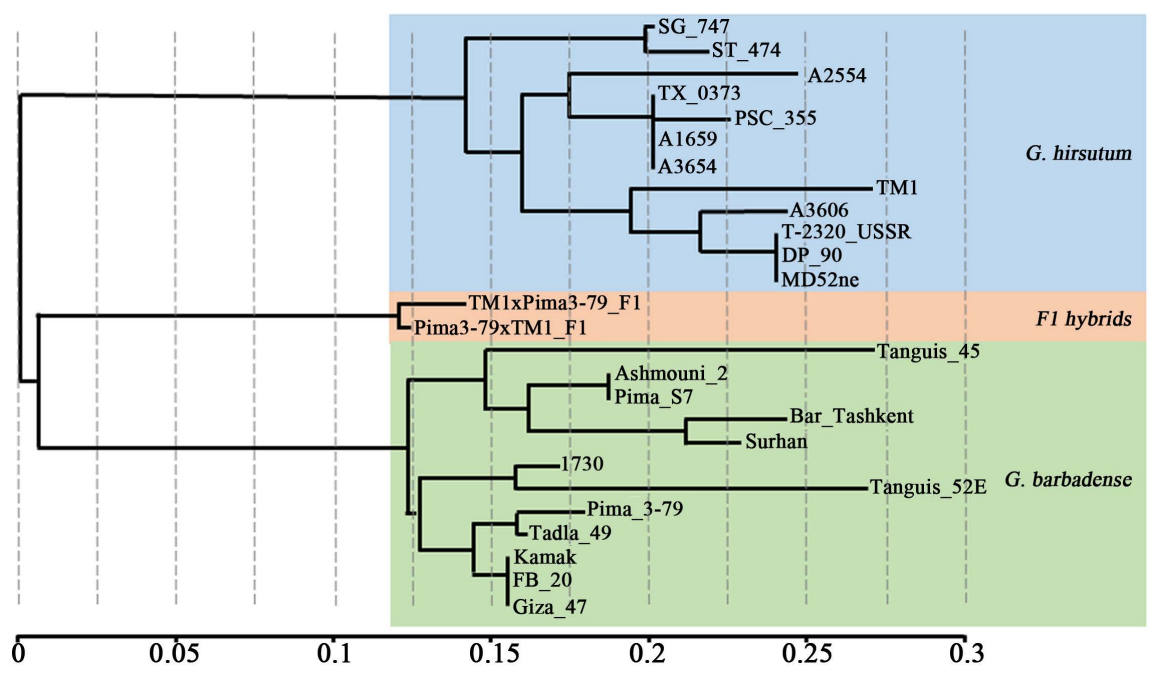

Figure 4. The dendrogram analysis of cotton fiber traits-associated SSR markers. The dendrogram of a set of improved Gossypium hirsutum and G. barbadense cotton lines constructed using JMP Genomics program on the basis of association of fiber traits with seven chromosome 25 specific SSR markers (Table S2). The results suggested that chromosome 25 is distinctly different between $G$. hirsutum and $G$. barbadense in fiber traits and molecular markers. The scale is presented as the branch length distance. 

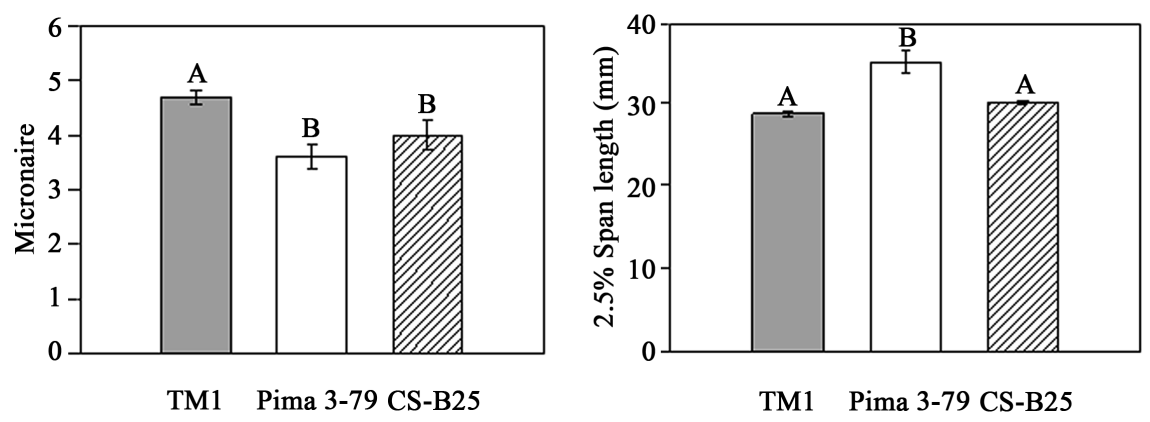

Figure 5. Comparison of micronaire and 2.5\% span length of CS-B25 fiber with TM-1 and Pima 3-79 lines. Some of the differentially expressed transcripts in CS-B25 might be associated with improved fiber quality traits. CS-B25 and TM-1 do not have significant differences in Fiber 2.5\% Span length, although CS-B25 had average values higher than TM-1. The letters marked above bars indicate statistically significant differences ( $\mathrm{p} \leq$ 0.005 in the micronaire analysis and $\mathrm{p} \leq 0.0005$ in the analysis of $2.5 \%$ span length). The error bars represent standard deviation of the mean.

Huang et al. [34] reported that AGPs are involved in fiber initiation and elongation. They reported that overexpression of a fasciclin-like arabinogalactan protein (GhFLA1) in G. hirsutum promoted fiber elongation and caused an increase in fiber length. In contrast, the suppression of GhFLA1 expression slowed down fiber initiation and elongation and produced shorter mature fiber. In addition, expression levels of GhFLAs and the genes related to primary cell wall biosynthesis were notably enhanced in the GhFLA1 overexpression transgenic fibers, whereas the transcripts of these genes were dramatically reduced in the fibers of GhFLA1 RNAi cotton plants. Using immunostaining methods, Huang et al. [34] further showed that both AGP composition and primary cell wall composition were changed in the transgenic fibers. The CS-B25 had several different differentially expressed AGP-like transcripts upregulated (Table S3), suggesting a major role of these genes at the fiber elongation stage. Previous studies revealed that the CS-B25 had several improved fiber quality traits including fiber length, strength, and micronaire [6] [7] [8] [9] [10]. These results were further confirmed from the top crosses with the selected USA improved cultivars that CS-B25 had several beneficial alleles with additive gene effects for improving fiber strength and micronaire in Upland cultivars [9] [10]. Our results suggest that the improvement of fiber traits in CS-B 25 line was in part associated with very high upregulation of GhFLA genes in fiber cell due to the introgression of the substituted chromosome of Pima 3-79 into the CS-B25 line. The dendrogram results based on the SSR markers also separated all lines into two broad groups of G. hirsutum and G. barbadense. It will be interesting in future to investigate the relation of differentially expressed transcripts of fasciclin-like arabinogalactan proteins among the lines of these two species with reference to the improved fiber traits including elongation. This is important considering that fiber elongation is one of the most important economic traits for determining the fiber price in global textile market. 
A large scale analysis of fiber gene expression in the elongation step had been previously conducted via cDNA array [48] [49] and deep RNA sequencing [50] [51]. Ji et al. [48] used subtractive PCR and cDNA macroarrays to identify 172 genes that were up-regulated in 10-DPA elongating fibers. They found that genes encoding a V-ATPase catalytic subunit, arabinogalactan proteins (AGPs), a kinesin-like calmodulin binding protein, cytochrome $\mathrm{P} 450$-like protein I, and proline-rich protein 5 were up-regulated. They also found that the mRNAs for proteins involved in signal transduction, lipid metabolism (such as LTP and ketoacyl CoA synthase), and cell wall-loosening enzymes (xyloglucan endotransglycosylase XET and expansins) were abundant. Using microarray analysis, Shi et al. [49] found that the ethylene biosynthesis pathway was up-regulated during fiber elongation, indicating that ethylene plays a role in promoting fiber elongation. Via high-throughput RNA sequencing, Wang et al. [50] conducted a global analysis of transcriptomes of two cotton lines, a wild type and a fuzzless/lintless mutant, at the early fiber development. They identified many genes differentially expressed in 2-8 DPA ovules, which included genes for a long-chain fatty acid elongation enzyme, 3-ketoacyl-CoA synthase, an ACC oxidase, NAC domain protein NAC2, P450 monooxygenase, WRKY transcription factor 45, MYB19, pectin esterase, chitinase, lipid transfer protein, and tubulin $\beta$-1 chain. Transcriptome analysis of short fiber mutant ligon lintless- 1 had shown that DEGs involved in very-long-chain fatty acid biosynthesis and cell wall metabolism were differentially expressed in fiber bearing ovules at 3 and 8 DPA [51]. In this study, the identified DEGs in 10-DPA CS-B25 fiber were very similar to those found by Ji et al. [48], Wang et al. [50], and Liang et al. [51]. We found that many genes involved in primary cell wall synthesis were up-regulated in CS-B25 during the fiber elongation stage, which included fasciclin-like arabinogalactan proteins [34], non-specific lipid transfer proteins, cellulose synthase, UDP-glycosyltransferase, pectin methylesterases, xyloglucan endotransglucosylase (XET), glycine-rich cell wall structural proteins, cytosolic pyruvate kinase [46], and ascorbate peroxidase [47]. Pectin methylesterases (PME), a multigene family, catalyze the demethylesterification of galacturonic acid units in pectins, which are the major components of plant cell wall. PME have been shown to play an important role in plant cell wall extension during stem elongation, pollen germination and pollen tube growth, and root development [52]. XETs are generally considered to be important enzymes in cell wall loosing, but they have been also shown to function in secondary cell wall genesis during the expansion process [53] [54]. Both PME and XET were found to be up-regulated in a cotton MD52ne line which had high fiber strength [55]. Several cotton non-specific lipid transfer protein genes, including $p F S 6, L T P 3, L T P 12$, were highly expressed in fiber cells [56] [57] [58]. LTP proteins have been proposed to participate in lipid transport for primary cell wall synthesis in fiber cells [59]. Recently, the functional role of cytosolic pyruvate kinase and ascorbate peroxidase in fiber elongation was elucidated. Zhang and Liu [46] showed that a cotton GhPK6 gene encoding a cytosolic pyruvate kinase was preferentially expressed in fibers, 
and its transcript level increased during the fiber elongation stage from 5 to 20 DPA and reached the maximum at the late elongation stage (20 DPA). Pyruvate kinase catalyzes the conversion of PEP to pyruvate in glycolysis, and pyruvate then undergoes oxidative phosphorylation in TCA cycle to generate the by-product, $\mathrm{H}_{2} \mathrm{O}_{2}$, a form of reactive oxygen species (ROS) which was also found to have elevated levels during fiber elongation [46]. The GhPK6 activity was negatively correlated with the rate of fiber elongation [46]. An ascorbate peroxidase, a reactive oxygen species (ROS) scavenging enzyme encoded by the GhAPX1 gene, was shown to be up-regulated in 10-DPA fibers [47]. Both the cytosolic pyruvate kinase and ascorbate peroxidase were involved in fiber elongation regulation in a ROS-mediated manner by modulating ROS homeostasis. A tubulin protein of tubulin beta 8 (Gh_Sca012883G01) and an ABI-1 like protein (Gh_A08G1131) in the assembly of cytoskeleton were also up-regulated (Table 3 and Table S3). It had been suggested that specific tubulins might affect microtubule organization and microfibril deposition in elongating fiber cells [37]. Three LRR receptor-like serine/threonine-protein kinases (Gh_D10G2231, Gh_D12G0597, and Gh_D02G0280) were highly up-regulated in CS-B25 (Table 3 and Table S3). It had been reported that these membrane-associated kinases were involved in signal transduction pathways [39], participating in cellulose deposition [40] and maintenance of cell wall integrity [41]. An LRR receptor-like kinase GhRLK1 was reported to be involved in secondary cell wall formation during fiber development [60]. Seventy-nine genes encoding Catharanthus roseus receptor-like kinases (CrRLK1Ls) had been identified in G. hirsutum TM-1 line. Some of them were found to be up-regulated in cotton fiber of chromosomal segment introgression lines (CSILs) with excellent fiber traits during the elongation stages from 10 to 20 DPA, but down-regulated at the early (5-DPA) and later (25-DPA) stages of fiber elongation. These observations revealed that RLKs positively affected fiber development [61].

The results of transcriptome analysis of 10 DPA fibers of the CS-B25 and TM-1 lines indicated that the synthesis and signaling pathways of GA, ethylene and auxin increased in CS-B25 when compared with TM-1. Many publications have indicated that ethylene modulates the elongation of cotton fiber cells. In this study, genes encoding the enzyme for ethylene synthesis,

1-aminocyclopropane-1-carboxylate oxidase (ACCO) and ethylene responsive transcription factors are up-regulated. Genes coding for ethylene overproduction 1-like protein (in chromatin remodeling and transcription regulator) and NAC transcription factors were also up-regulated. The gene encoding a RING E3 ligase XBAT32 (Gh_A08G0780), which targets the ACS proteins, was down-regulated in the CS-B25 fiber. This down regulation resulted in a slight increase of the ACS level. Auxin has been shown to promote fiber initiation and elongation and play an important role in fiber development [26]. The transcript levels of auxin-responsive GH3 protein, auxin-responsive IAA14 protein, and auxin influx carrier family protein in CS-B25 10 DPA fiber were up-regulated, suggesting that they may have important functions in the fiber elongation. 
Several genes encoding MYB transcription factors were found to be highly up-regulated in the CS-B25 elongating fibers. A total of 524 non-redundant MYB genes had been identified in the upland cotton genome [62]. Among them, the G. hirsutum MYB genes MYB2, MYB3, MYB5, MYB7, MYB20, MYB25, MYB42, MYB43, MYB46, MYB52, MYB54, MYB69, MYB85, MYB103, and $M Y B 109$ were shown to be highly expressed in fiber [42] [62], suggesting that they play important roles in fiber development. GhMYB109 was reported to participate in fiber development, and the suppression of its expression resulted in the reduction of fiber length [63]. GhMYB25 was shown to regulate trichome formation and act as a key factor in early cotton fiber development [64]. We had previously reported that a cotton MYB gene GhMYB7 was differentially expressed in developing fibers, and GhMYB7 was involved in transcriptional regulation of a LTP3 gene encoding lipid transfer protein [65]. The GhMYB7 was later shown to activate genes involved in secondary cell wall thickening and biosynthesis [66]. Besides the MYB transcription factors, homeobox leucine zipper (HD-Zip) transcription factors are also up-regulated in CS-B25. HD-Zip proteins were shown to play important roles in cotton fiber differentiation [67] [68]. Many NAC transcription factors were also highly expressed in CS-B25 10-DPA fibers. A total of 143 and 145 NAC genes have been identified in the genomes of the two diploid cottons $G$. arboretum and G. raimondii, respectively [69]. Among them, more than 30 had been shown to have higher expression levels in 10 - 20 DPA fiber. NAC genes had been shown to control secondary cell wall thickening during fiber development [69]. Our transcript profile observations during fiber development are similar to those of Al-Ghazi et al. [70]. Similar results had also been observed in some of Upland cotton chromosome introgression lines (CSILs) carrying different G. barbadense chromosomal segments in the recurrent parent TM-1 [71] [72]. These CSIL lines with longer and stronger fiber had increased expression in genes involved in cell wall biosynthesis, hormone signal transduction, and in genes encoding transcriptions factors during the fiber elongation stage. Both CS-B25 and chromosome segment introgression lines (CSILs) were useful resources for transcriptome profiling to study molecular mechanism of positive fiber traits.

Genome wide analysis has identified 116 and 102 WRKY genes in G. ramondii and G. hirsutum, respectively [73]. We have only found that two WRKY transcription factors, WRKY 21 and WRKY 29, were moderately up-regulated in CS-B25. This observation is consistent with the results of Dou et al. [73], in that many GhWRKY transcription factors had high expression levels at 0, 20, and 25 DPA fiber and they are involved in fiber initiation and secondary wall synthesis. Using the homologous alignment method, 143 genes for NAC-transcription factors in the G. arboretum genome were identified [69]. Many of them were highly expressed in 15 DPA fiber cells and might be involved in the fiber secondary wall thickening.

The genetic effects in the CS-B25 line could be due to any of the following reasons: 1) the genes on the substituted chromosome, 2) the genes on the re- 
maining chromosomes of its recurrent parent, and 3) an interaction between the gene(s) on the substituted chromosome and the remaining chromosomes of its recurrent parent [8]. A comparative analysis of CS-B25 with TM-1 is expected to show the differential transcripts from the direct effect of alien species substituted chromosome or an interaction effect of the substituted chromosome and the remaining chromosomes of CS-B25. Results showed that the majority genes upand down-regulated in 10-DPA fiber in CS-B25 were not located on Pima 3-79 chromosome 25, which is mapped to Gh_D06G in the G. hirsutum (AD1) NAU-NBI genome used in this study (Table S3) [74]. This observation suggested that chromosome interactions between the substituted $G$. barbadense chromosome 25 pair and other non-substituted chromosomes of TM-1 might be involved in potential gene regulation. This is important considering that molecular markers (including EST markers) are used to construct linkage map of important QTLs with the expectation that these markers are directly associated with the QTL of interest in the same chromosomal region and can be used in marker assisted selection using genomic DNA to expedite the breeding program. However, our results showed that some of the ESTs may not have direct association with the chromosome but can cause a chromosomal effect on the genes. For example, the substituted $G$. barbadense chromosome 25 may contain potential regulatory elements that interact with gene located on other chromosomes in CS-B25 to cause an effect. Our results showed that several transcription factor like elements were associated with differentially expressed transcripts in CS-B25 line. It would be interesting in future investigation to identify the roles of some inter-chromosomal interactions in the activation or repression of gene transcription. The role of long-range chromosomal interactions involved in gene regulation has been reviewed in details by different research groups [75] [76]. The CS-B25 line can be used as a tool for the study of cotton chromosomal interactions and their role in transcriptional regulation because of the unique genetic background of CS-B25. The Chromosome Conformation Capture (3C), a high throughput technique developed by Dekker et al. [77], has been widely used to study chromosomal conformation/interaction. Using the 3C-based technology would allow us to study chromosomal interactions in CS-B25 and reveal how the interactions might affect up or down regulation of gene expression.

The results from the dendrogram and fiber QTL analyses suggested that chromosome 25 from $G$. barbadense is associated with potential valuable fiber traits (Figure 4). A previous report [8] also documented that CS-B25 had important association with several improved fiber traits, including decreasing micronaire, increasing fiber length and strength compared to TM-1 and some USA commercial cultivars, suggesting that CS-B25 would be an important genetic tool to discover many useful genes associated with fiber development. Cotton fiber, an elongated and thickened single cell of the seed epidermis in which the fiber cell wall polymers help to determine primarily the cotton fiber quality. Cotton fiber cell undergoes several phase of changes since its initiation: 1) extreme elongation (to $>2.5 \mathrm{~cm}$ ) via primary wall synthesis till about 10 days; 2) then 
transitional wall thickening and primary wall remodeling; and 3) secondary wall thickening via deposition of nearly pure cellulose; and 4) final maturation and cell death processes before boll opening to reveal the fluffy fiber within the cotton bolls [78]. Our results showed that CS-B25 had several up or down regulated differentially expressed transcripts in 10 DPA fiber associated with potential cytoskeleton or cell wall genes (Table 3). It is important to note that most of the improved cotton fiber quality traits are primarily controlled by the cytoskeleton or cell wall related genes during fiber development [77]. These results suggested that future investigation with these differentially expressed transcripts, especially very high upregulated expression of fasciclin-like arabinogalactan protein 7 genes in CS-B25 10 DPA fiber cell, will have great potential to elucidate genes associated with fiber quality traits at the molecular level.

\section{Conclusion}

This study showed that the CS-B25 line is a valuable genetic resource to discern many novel differentially expressed genes due to the effect of the substituted alien species chromosome 25 or its interaction with other chromosomes in CS-B25. The CS-B25 line had several valuable fiber quality traits including micronaire and fiber length associated with the substituted chromosome 25 from G. barbadense. We discovered that several genes involved in hormone signal transduction, cell wall synthesis, and transcription factors in regulating cell wall synthesis are up-regulated in 10 DPA fiber of the CS-B25 line. These differentially expressed genes could be used as potential resources for fiber quality improvement. This research will facilitate our understanding of molecular mechanisms of fiber cell elongation and will allow us to develop new approaches for cotton fiber improvement.

\section{Acknowledgements}

The authors gratefully acknowledge that this research was made possible through the collaborative efforts between USDA/ARS and Mississippi State University/Mississippi Agriculture and Forestry Experimental Station (MAFES). We thank Uzbek government in providing training opportunities for Mr. Mirazakamol A. Ayubov in the USA laboratories at USDA and Mississippi State University, Mississippi State, Mississippi.

\section{References}

[1] Basra, A.S. and Malik, C. (1984) Development of the Cotton Fiber. International Review of Cytology, 89, 65-113. https://doi.org/10.1016/S0074-7696(08)61300-5

[2] DeLanghe, E.A.L. (1986) Lint Development. In: Mauney, J.R. and Stewart, J.M., Ed., Cotton Physiology, The Cotton Foundation, Memphis, 325-349.

[3] http://cottonaustralia.com.au/cotton-library/fact-sheets/cotton-fact-file-cotton-pro perties-and-products

[4] Tyagi, P., Gore, M.A., Bowman, D.T., Campbell, B.T., Udall, J.A. and Kuraparthy, V. (2014) Genetic Diversity and Population Structure in the US Upland Cotton 
(Gossypium hirsutum L.). Theoretical and Applied Genetics, 127, 283-295. https://doi.org/10.1007/s00122-013-2217-3

[5] Stelly, D.M., Saha, S., Raska, D.A., Jenkins, J.N., McCarty, J.C. and Gutierres, O.A. (2005) Registration of 17 Upland (Gossypium hirsutum) Cotton Germplasm Lines Disomic for Different G. barbadense Chromosome or Arm Substitutions. Crop Science, 45, 2663-2665. https://doi.org/10.2135/cropsci2004.0642

[6] Saha, S., Wu, J., Jenkins, J.N., McCarty, J.C., Stelly, D.M., Percy, R.G., Raska, D.A. and Gutierrez, O.A. (2004) Effect of Chromosome Substitutions from Gossypium barbadense L. 3-79 into G. hirsutum L. TM-1 on Agronomic and Fiber Traits. The Journal of Cotton Science, 8, 162-169.

[7] Saha, S., Jenkins, J.N., Wu, J., McCarty, J.C., Gutierrez, O.A., Percy, R.G., Cantrell, R.G. and Stelly, D.M. (2006) Effects of Chromosome-Specific Introgression in Upland Cotton on Fiber and Agronomic Traits. Genetics, 172, 1927-1938.

https://doi.org/10.1534/genetics.105.053371

[8] Wu, J., Jenkins, J.N., McCarty, J.C., Saha, S. and Stelly, D.M. (2006) An Additive Dominance Model to Determine Chromosomal Effects in Chromosome Substitution Lines and Other Germplasms. Theoretical and Applied Genetics, 112, 391-399. https://doi.org/10.1007/s00122-005-0042-Z

[9] Jenkins, J.N., McCarty, J.C., Wu, J., Saha, S., Gutierrez, O.A., Hayes, R. and Stelly, D.M. (2007) Genetic Effects of Thirteen Gossypium barbadense L. Chromosome Substitution Lines in Top Crosses with Upland Cotton Cultivars: II. Fiber Quality Traits. Crop Science, 47, 561-570. https://doi.org/10.2135/cropsci2006.06.0396

[10] Wu, J., Jenkins, J.N., McCarty, J.C. and Saha, S. (2010) Genetic Effects of Individual Chromosomes in Cotton Cultivars Detected by Using Chromosome Substitution Lines as Genetic Probes. Genetica, 138, 1171-1179. https://doi.org/10.1007/s10709-010-9507-3

[11] Saha, S., Wu, J., Jenkins, J.N., McCarty, J., Hayes, R. and Stelly, D.M. (2010) Genetic Dissection of Chromosome Substitution Lines Discovered Novel Alleles in Gossypium barbadense L. with Potential for Improving Agronomic Traits Including Yield. Theoretical and Applied Genetics, 120, 1193-1205. https://doi.org/10.1007/s00122-009-1247-3

[12] Wu, Z., Soliman, K.M., Bolton, J.J., Saha, S. and Jenkins, J.N. (2008) Identification of Differentially Expressed Genes Associated with Cotton Fiber Development in a Chromosomal Substitution Line (CS-B22sh). Functional \& Integrative Genomics, 8, 165-174. https://doi.org/10.1007/s10142-007-0064-5

[13] Wan, C.Y. and Wilkins, T.A. (1994) A Modified Hot Borate Method Significantly Enhances the Yield of High-Quality RNA from Cotton (Gossypium hirsutum L.). Analytical Biochemistry, 223, 7-12. https://doi.org/10.1006/abio.1994.1538

[14] Zhang, T., Hu, Y., Jiang, W., Fang, L., Guan, X., Chen, J., Zhang, J., Saski, C.A., Scheffler, B.E., Stelly, D.M., et al. (2015) Sequencing of Allotetraploid Cotton (Gossypium hirsutum L. acc. TM-1) Provides a Resource for Fiber Improvement. Nature Biotechnology, 33, 531-537. https://doi.org/10.1038/nbt.3207

[15] Kim, D., Pertea, G., Trapnell, C., Pimentel, H., Kelley, R. and Salzberg, S.L. (2013) TopHat2: Accurate Alignment of Transcriptomes in the Presence of Insertions, Deletions and Gene Fusions. Genome Biology, 14, R36.

https://doi.org/10.1186/gb-2013-14-4-r36

[16] Anders, S., Pyl, P.T. and Huber, W. (2015) HTSeq-A Python Framework to Work with High-Throughput Sequencing Data. Bioinformatics, 31, 166-169. https://doi.org/10.1093/bioinformatics/btu638 
[17] Robinson, M.D., McCarthy, D.J. and Smyth, G.K. (2010) EdgeR: A Bioconductor Package for Differential Expression Analysis of Digital Gene Expression Data. Bioinformatics, 26, 139-140. https://doi.org/10.1093/bioinformatics/btp616

[18] Luo, W., Friedman, M.S., Shedden, K., Hankenson, K.D. and Woolf, J.P. (2009) GAGE: Generally Applicable Gene Set Enrichment for Pathway Analysis. BMC Bioinformatics, 10, 161. https://doi.org/10.1186/1471-2105-10-161

[19] Ashburner, M., Ball, C.A., Blake, J.A., Botstein, D., Butler, H., Cherry, J.M., Davis, A.P., Dolinski, K., Dwight, S.S., Eppig, J.T., Harris, M.A., Hill, D.P., Issel-Tarver, L., Kasarskis, A., Lewis, S., Matese, J.C., Richardson, J.E., Ringwald, M., Rubin, G.M. and Sherlock, G. (2000) Gene Ontology: Tool for the Unification of Biology. Nature Genetics, 25, 25-29. https://doi.org/10.1038/75556

[20] Ogata, H., Goto, S., Sato, K., Fujibuchi, W., Bono, H. and Kanehisa, M. (1999) KEGG: Kyoto Encyclopedia of Genes and Genomes. Nucleic Acids Research, 27, 29-34. https://doi.org/10.1093/nar/27.1.29

[21] Yu, J., Jung, S., Cheng, C.-H., Ficklin, S.P., Lee, T., Zheng, P., Jones, D., Percy, R.G. and Main, D. (2013) CottonGen: A Genomics, Genetics and Breeding Database for Cotton Research. Nucleic Acids Research, 42, D1229-D1236.

https://doi.org/10.1093/nar/gkt1064

[22] Supek, F., Bosnjak, M., Skunca, N. and Smuc, T. (2011) REVIGO Summarizes and Visualizes Long Lists of Gene Ontology Terms. PLoS ONE, 6, e21800. https://doi.org/10.1371/journal.pone.0021800

[23] Sun, Q., Jiang, H., Zhu, X., Wang, W., He, X., Shi, Y., Yuan, Y., Du, X. and Cai, Y. (2013) Analysis of Sea-Island and Upland Cotton in Response to Verticillium dahlia Infection by RNA Sequencing. BMC Genomics, 14, 852. https://doi.org/10.1186/1471-2164-14-852

[24] Livak, K.J. and Schmittgen, T.D. (2001) Analysis of Relative Gene Expression Data Using Real-Time Quantitative PCR and the $2^{-\Delta \triangle C T}$ Method. Methods, 25, 402-408. https://doi.org/10.1006/meth.2001.1262

[25] Egamberdiev, S., Saha, S., Salakhutdinov, I., Jenkins, J.N., Deng, D., Khurshut, E. and Abdurakhmonov, I. (2016) Comparative Assessment of Genetic Diversity in Cytoplasmic and Nuclear Genome of Upland Cotton. Genetica, 144, 289-306. https://doi.org/10.1007/s10709-016-9898-x

[26] Liao, W., Zhang, J., Xu, N. and Peng, M. (2010) The Role of Phytohormones in Cotton Fiber Development. Russian Journal of Plant Physiology, 57, 462-468. https://doi.org/10.1134/S1021443710040023

[27] Ruduś, I., Sasiak, M. and kepczyński, J. (2013) Regulation of Ethylene Biosynthesis at the Level of 1-Aminocyclopropane-1-Carboxylate Oxidase (ACO) Gene. Acta Physiologiae Plantarum, 35, 295-307. https://doi.org/10.1007/s11738-012-1096-6

[28] Qin, Y.-M., Hu, C.-Y., Pang, Y., Kastaniotis, A.J., Hiltunen, J.K. and Zhu, Y.-X. (2007) Saturated Very-Long Fatty Acids Promote Cotton Fiber and Arabidopsis Cell Elongation by Activating Ethylene Biosynthesis. Plant Cell, 19, 3692-3704. https://doi.org/10.1105/tpc.107.054437

[29] Kim, H.-S., Kim, S.J., Abbasi, N., Bressan, R.A., Yun, D.-J., Yoo, S.-D., Kwon, S.-Y. and Choi, S.-B. (2010) The DOF Transcription Factor Dof5.1 Influences Leaf Axial Patterning by Promoting Revoluta Transcription in Arabidopsis. The Plant Journal, 64, 524-535. https://doi.org/10.1111/j.1365-313X.2010.04346.x

[30] Prasad, M.E., Schofield, A., Lyzenga, W., Liu, H. and Stone, S. (2010) Arabidopsis RING E3 Ligase XBAT32 Regulates Lateral Root Production through Its Role in Ethylene Biosynthesis. Plant Physiology, 153, 1587-1596. 
https://doi.org/10.1104/pp.110.156976

[31] Pech, J.C., Latché, A. and Bouzayen, M. (2010) Ethylene Biosynthesis. In: Davies, P.J., Ed., Plant Hormones: Biosynthesis, Signal Transduction, Action, 3rd Edition, Springer, Dordrecht, 115-136. https://doi.org/10.1007/978-1-4020-2686-7_6

[32] Gosti, F., Beaudoin, N., Serizet, C., Webb, A.A.R., Vartanian, N. and Giraudat, J. (1999) ABI1 Protein Phosphatase 2C Is a Negative Regulator of Abscisic Acid Signaling. Plant Cell, 11, 1897-1909. https://doi.org/10.1105/tpc.11.10.1897

[33] Gilbert, M.K., Bland, J.M., Shockey, J.M., Cao, H., Hinchliffe, D.J., Fang, D.D. and Naoumkina, M. (2013) A Transcript Profiling Approach Reveals an Abscisic Acid-Specific Glycosyltransferase (UGT73C14) Induced in Developing Fiber of Ligon Lintless-2 Mutant of Cotton (Gossypium hirsutum L.). PLoS ONE, 8, e75268. https://doi.org/10.1371/journal.pone.0075268

[34] Huang, G.-Q., Gong, S.-Y., Xu, W.-L., Li, W., Li, P., Zhang, C.-J., Li, D.-D., Zheng, Y., Li, F.-G. and Li, X.-B. (2013) A Fasciclin-Like Arabinogalactan Protein, GhFLA1, Is Involved in Fiber Initiation and Elongation of Cotton. Plant Physiology, 161, 1278-1290. https://doi.org/10.1104/pp.112.203760

[35] Deng, T., Yao, H., Wang, J., Wang, J., Xue, H. and Zuo, K. (2016) GhLTPG1, a Cotton GPI-Anchored Lipid Transfer Protein, Regulates the Transport of Phosphatidylinositol Monophosphates and Cotton Fiber Elongation. Scientific Reports, 6, Article No. 26829.

[36] Liu, Q., Talbot, M. and Llewellyn, D.J. (2013) Pectin Methylesterase and Pectin Remodeling Differ in the Fibre Walls of Two Gossypium Species with Different Fibre Properties. PLOS ONE, 8, e65131. https://doi.org/10.1371/journal.pone.0065131

[37] Whittaker, D.J. and Triplett, B.A. (1999) Gene Specific Changes in $\alpha$-Tubulin Transcript Accumulation in Developing Cotton Fibers. Plant Physiology, 121, 181-188. https://doi.org/10.1104/pp.121.1.181

[38] Szymanski, D.B. (2005) Breaking the WAVE Complex: The Point of Arabidopsis Trichomes. Current Opinion in Plant Biology, 8, 103-112. https://doi.org/10.1016/j.pbi.2004.11.004

[39] Afzal, A.J., Wood, A.J. and Lightfoot, D.A. (2008) Plant Receptor-Like Serine Threonine Kinases: Roles in Signaling and Plant Defense. Molecular Plant-Microbe Interactions, 21, 507-517. https://doi.org/10.1094/MPMI-21-5-0507

[40] Xu, S.-L., Rahman, A., Baskin, T.I. and Kieber, J.J. (2008) Two Leucine-Rich Repeat Receptor Kinases Mediate Signaling, Linking Cell Wall Biosynthesis and ACC Synthase in Arabidopsis. Plant Cell, 20, 3065-3079. https://doi.org/10.1105/tpc.108.063354

[41] Hamann, T. (2015) The Plant Cell Wall Integrity Maintenance Mechanism-A Case Study of a Cell Wall Plasma Membrane Signaling Network. Phytochemistry, 112, 100-109. https://doi.org/10.1016/j.phytochem.2014.09.019

[42] Zhong, Z., Lee, C., Zhou, J., McCarthy, R.L. and Ye, Z.-H. (2008) A Battery of Transcription Factors Involved in the Regulation of Secondary Cell Wall Biosynthesis in Arabidopsis. Plant Cell, 20, 2763-2782. https://doi.org/10.1105/tpc.108.061325

[43] Ko, J.-H., Kim, W.-C., Kim, J.-Y., Ahn, S.-J. and Han, K.-H. (2012) MYB46-Mediated Transcriptional Regulation of Secondary Wall Synthesis. Molecular Plant, 5, 961-963. https://doi.org/10.1093/mp/sss076

[44] Zhong, R. and Ye, Z.H. (2012) MYB46 and MYB83 Bind to the SMRE Sites and Directly Activate a Suite of Transcription Factors and Secondary Wall Biosynthetic Genes. Plant and Cell Physiology, 53, 368-380. https://doi.org/10.1093/pcp/pcr185 
[45] Shang, H., Li, W., Zou, C. and Yuan, Y. (2013) Analyses of the NAC Transcription Factor Gene Family in Gossypium raimondii Ulbr: Chromosomal Location, Structure, Phylogeny, and Expression Patterns. Journal of Integrative Plant Biology, 55, 663-676. https://doi.org/10.1111/jipb.12085

[46] Zhang, B. and Liu, J.Y. (2016) Cotton Cytosolic Pyruvate Kinase GhPK6 Participates in Fast Fiber Elongation in a ROS-Mediated Manner. Planta, 244, 915-926. https://doi.org/10.1007/s00425-016-2557-8

[47] Guo, K., Du, X., Tu, L., Tang, W., Wang, P., Wang, M., Liu, Z. and Zhang, X. (2016) Fibre Elongation Requires Normal Redox Homeostasis Modulated by Cytosolic Ascorbate Peroxidase in Cotton (Gossypium hirsutum). Journal of Experimental Botany, 67, 3289-3301. https://doi.org/10.1093/jxb/erw146

[48] Ji, S.-J., Lu, Y.-C., Feng, J.-X., Wei, G., Li, J., Shi, Y.-H., Fu, Q., Liu, D., Luo, J.-C. and Zhu, Y.-X. (2003) Isolation and Analysis of Genes Preferentially Expressed during Early Cotton Fiber Development by Subtractive PCR and cDNA Array. Nucleic Acids Research, 31, 2534-2543. https://doi.org/10.1093/nar/gkg358

[49] Shi, Y.H., Zhu, S.W., Mao, X.Z., Feng, J.X., Qin, Y.M., Zhang, L., et al. (2006) Transcriptome Profiling, Molecular Biological, and Physiological Studies Reveal a Major Role for Ethylene in Cotton Fiber Elongation. Plant Cell, 18, 651-664. https://doi.org/10.1105/tpc.105.040303

[50] Wang, Q.Q., Liu, F., Chen, X.S., Ma, X.J., Zeng, H.Q. and Yang, Z.M. (2010) Transcriptome Profiling of Early Developing Cotton Fiber by Deep-Sequencing Reveals Significantly Differential Expression of Genes in a Fuzzless/Lintless Mutant. Genomics, 96, 369-376. https://doi.org/10.1016/j.ygeno.2010.08.009

[51] Liang, W., Fang, L., Xiang, D., Hu, Y., Feng, H., Chang, L. and Zhang, T. (2015) Transcriptome Analysis of Short Fiber Mutant Ligon Lintless-1 (Li1) Reveals Critical Genes and Key Pathways in Cotton Fiber Elongation and Leaf Development. PLoS ONE, 10, e0143503. https://doi.org/10.1371/journal.pone.0143503

[52] Micheli, F. (2001) Pectin Methylesterases: Cell Wall Enzymes with Important Roles in Plant Physiology. Trends in Plant Science, 6, 414-419. https://doi.org/10.1016/S1360-1385(01)02045-3

[53] Bourquin, V., Nishikubo, N., Abe, H., Brumer, H., Denman, S., Eklund, M., Christiernin, M., Teeri, T.T., Sundberg, B. and Mellerowicz, E.J. (2002) Xyloglucan Endotransglycosylases Have a Function during the Formation of Secondary Cell Walls of Vascular Tissues. Plant Cell, 14, 3073-3088. https://doi.org/10.1105/tpc.007773

[54] Lee, J., Burns, T.H., Light, G., Sun, Y., Fokar, M., Kasukabe, Y., et al. (2010) Xyloglucan Endotransglycosylase/Hydrolase Genes in Cotton and Their Role in Fiber Elongation. Planta, 232, 1191-1205. https://doi.org/10.1007/s00425-010-1246-2

[55] Islam, M.S., Fang, D.D., Thyssen, G.N., Delhom, C.D., Liu, Y. and Kim, H.J. (2016) Comparative Fiber Property and Transcriptome Analyses Reveal Key Genes Potentially Related to High Fiber Strength in Cotton (Gossypium hirsutum L.) Line MD52ne. BMC Plant Biology, 16, 36. https://doi.org/10.1186/s12870-016-0727-2

[56] Orford, S.J. and Timmis, J.N. (2000) Expression of a Lipid Transfer Protein Gene Family during Cotton Fibre Development. Biochimica et Biophysica Acta, 1483, 275-284. https://doi.org/10.1016/S1388-1981(99)00194-8

[57] Hsu, C.Y., Creech, R.G., Jenkins, J.N. and Ma, D.P. (1999) Analysis of Promoter Activity of Cotton Lipid Transfer Protein Gene LTP6 in Transgenic Tobacco Plants. Plant Science, 143, 63-70. https://doi.org/10.1016/S0168-9452(99)00026-6

[58] Liu, H.C., Creech, R.G., Jenkins, J.N. and Ma, D.P. (2000) Cloning and Promoter Analysis of the Cotton Lipid Transfer Protein Gene Ltp3. Biochimica et Biophysica 
Acta, 1487, 106-111. https://doi.org/10.1016/S1388-1981(00)00072-X

[59] Ma, D.P., Liu, H.C., Tan, H., Creech, R.G., Jenkins, J.N. and Chang, Y.F. (1997) Cloning and Characterization of a Lipid Transfer Protein Gene Specifically Expressed in Fiber Cells. Biochimica et Biophysica Acta, 1344, 111-114. https://doi.org/10.1016/S0005-2760(96)00166-X

[60] Li, Y.-L., Sun, J. and Xia, G.-X. (2005) Cloning and Characterization of a Gene for an LRR Receptor-Like Protein Kinase Associated with Cotton Fiber Development. Molecular Genetics and Genomics, 273, 217-224. https://doi.org/10.1007/s00438-005-1115-Z

[61] Niu, E., Cai, C., Zheng, Y., Shang, X. and Fang, L. (2016) Genome-Wide Analysis of CrRLK1L Gene Family in Gossypium and Identification of Candidate CrRLK1L Genes Related to Fiber Development. Molecular Genetics and Genomics, 291, 1137-1154. https://doi.org/10.1007/s00438-016-1169-0

[62] Salih, H., Gong, W., He, S., Sun, G., Sun, J. and Du, X. (2016) Genome-Wide Characterization and Expression Analysis of MYB Transcription Factors in Gossypium hirsutum. BMC Genetics, 17, 129. https://doi.org/10.1186/s12863-016-0436-8

[63] Pu, L., Li, Q., Fan, X., Yang, W. and Xue, Y. (2008) The R2R3 MYB Transcription Factor GhMYB109 Is Required for Cotton Fiber Development. Genetics, 180, 811-820. https://doi.org/10.1534/genetics.108.093070

[64] Walford, S.A., Wu, Y., Ilewellyn, D.J. and Dennis, E.S. (2011) GhMYB25-Like: A Key Factor in Early Cotton Fibre Development. The Plant Journal, 65, 785-797. https://doi.org/10.1111/j.1365-313X.2010.04464.x

[65] Hsu, C.Y., Jenkins, J.N., Saha, S. and Ma, D.P. (2005) Transcriptional Regulation of the Lipid Transfer Protein Gene LTP3 in Cotton Fibers by a Novel MYB Protein. Plant Science, 168, 167-181. https://doi.org/10.1016/j.plantsci.2004.07.033

[66] Huang, J., Chen, F., Wu, S., Li, J. and Xu, W. (2016) Cotton GhMYB7 Is Predominantly Expressed in Developing Fibers and Regulates Secondary Cell Wall Biosynthesis in Transgenic Arabidopsis. Science China Life Sciences, 59, 194-205. https://doi.org/10.1007/s11427-015-4991-4

[67] Walford, S.A., Wu, Y., Ilewellyn, D.J. and Dennis, E.S. (2012) Epidermal Cell Differentiation in Cotton Mediated by the Homeodomain Leucine Zipper Gene, GhHD-1. The Plant Journal, 71, 464-478.

[68] Zahur, M., Asif, M.A., Zeeshan, N., Mehmood, S., Malik, M.F. and Asif, A.R. (2013) Homeobox Leucine Zipper Proteins and Cotton Improvement. Advances in Bioscience and Biotechnology, 4, 15-20. https://doi.org/10.4236/abb.2013.410A3003

[69] Shang, H., Wang, Z., Zou, C., Zhang, Z., Li, W., Li, J., Shi, Y., Gong, W., Chen, T., Liu, A., Gong, J., Ge, Q. and Yuan, Y. (2016) Comprehensive Analysis of NAC Transcription Factors in Diploid Gossypium: Sequence Conservation and Expression Analysis Uncover Their Roles during Fiber Development. Science China Life Sciences, 59, 142-153. https://doi.org/10.1007/s11427-016-5001-1

[70] Al-Ghazi, Y., Bourot, S., Arioli, T., Dennis, E.S. and Llewellyn, D.J. (2009) Transcript Profiling during Fiber Development Identifies Pathways in Secondary Metabolism and Cell Wall Structure That May Contribute to Cotton Fiber Quality. Plant and Cell Physiology, 50, 1364-1381. https://doi.org/10.1093/pcp/pcp084

[71] Fang, L., Tian, R., Chen, J., Wang, S., Li, X., Wang, P. and Zhang, T. (2014) Transcriptomic Analysis of Fiber Strength in Upland Cotton Chromosome Introgression Lines Carrying Different Gossypium barbadense Chromosomal Segments. PLoS ONE, 9, e94642. https://doi.org/10.1371/journal.pone.0094642

[72] Fang, L., Tian, R., Li, X., Chen, J., Wang, S., Wang, P. and Zhang, T. (2014) Cotton 
Fiber Elongation Network Revealed by Expression Profiling of Longer Fiber Lines Introgressed with Different Gossypium barbadense Chromosome Segments. BMC Genomics, 15, 838. https://doi.org/10.1186/1471-2164-15-838

[73] Dou, L., Zhang, X., Pang, C., Song, M., Wei, H., Fan, S. and Yu, S. (2014) Genome-Wide Analysis of the WRKY Gene Family in Cotton. Molecular Genetics and Genomics, 289, 1103-1121. https://doi.org/10.1007/s00438-014-0872-y

[74] Yu, J.Z., Ulloa, M., Hoffman, S.M., Kohel, R.J., Pepper, A.E., Fang, D.D., Percy, R.G. and Burke, J.J. (2014) Mapping Genomic Loci for Cotton Plant Architecture, Yield Components, and Fiber Properties in an Interspecific (Gossypium hirsutum L. x G. barbadense L.) RIL Population. Molecular Genetics and Genomics, 289, 1347-1367. https://doi.org/10.1007/s00438-014-0930-5

[75] Bartkuhn, M. and Renkawitz, R. (2008) Long Range Chromatin Interactions Involved in Gene Regulation. Biochimica et Biophysica Acta, 1783, 2161-2166. https://doi.org/10.1016/j.bbamcr.2008.07.011

[76] Miele, A. and Dekker, J. (2008) Long-Range Chromosomal Interactions and Gene Regulation. Molecular BioSystems, 4, 1046-1057. https://doi.org/10.1039/b803580f

[77] Dekker, J., Rippe, K., Dekker, M. and Kleckner, N. (2002) Capturing Chromosome Conformation. Science, 295, 1306-1311. https://doi.org/10.1126/science.1067799

[78] Haigler, C.H., Betancur, L., Stiff, M.R. and Tuttle, J.R. (2012) Cotton Fiber: A Powerful Single-Cell Model for Cell Wall and Cellulose Research. Front Plant Science, 3, 104. https://doi.org/10.3389/fpls.2012.00104

[79] Mei, H., Zhu, X., Guo, W., Cai, C. and Zhang, T. (2014) Exploitation of Chinese Upland Cotton Cultivar Germplasm Resources to Mine Favorable QTL Alleles Using Association Mapping. In: Abdurakhmonov, I.Y., Ed., World Cotton Germplasm Resources, Intech, Rijeka, 55-85.

[80] Zhang, Z., Li, J.W., Muhammad, J., Cai, J., Jia, F., Shi, Y.Z., et al. (2015) High Resolution Consensus Mapping of Quantitative Trait Loci for Fiber Strength, Length and Micronaire on Chromosome 25 of the Upland Cotton (Gossypium hirsutum L.). PLoS ONE, 10, e0135430. https://doi.org/10.1371/journal.pone.0135430

[81] Abdurakhmonov, I.Y., Saha, S., Jenkins, J.N., Buriev, Z.T., Shermatov, S.E., Scheffler, B.E., et al. (2009) Linkage Disequilibrium Based Association Mapping of Fiber Quality Traits in G. hirsutum L. Variety Germplasm. Genetica, 136, 401-417. https://doi.org/10.1007/s10709-008-9337-8

[82] Sun, F.D., Zhang, J.H., Wang, S.F., Gong, W.K., Shi, Y.Z., Liu, A.Y., Li, J.W., Gong, J.W., Shang, H.H. and Yuan, Y.L. (2012) QTL Mapping for Fiber Quality Traits across Multiple Generations and Environments in Upland Cotton. Molecular Breeding, 30, 569-582. https://doi.org/10.1007/s11032-011-9645-Z

[83] Liang, Q., Hu, C., Hua, H., Li, Z. and Hua, J. (2013) Construction of a Linkage Map and QTL Mapping for Fiber Quality Traits in Upland Cotton (Gossypium hirsutum L.). Chinese Science Bulletin, 58, 3233-3243. https://doi.org/10.1007/s11434-013-5807-1

[84] Qin, H., Chen, M., Yi, X., Bie, S., Zhang, C., Zhang, Y., et al. (2015) Identification of Associated SSR Markers for Yield Component and Fiber Quality Traits Based on Frame Map and Upland Cotton Collections. PLOS ONE, 10, e0118073. https://doi.org/10.1371/journal.pone.0118073 


\section{Supplement}

Table S1. Primers used for quantitative RT-PCR.

\begin{tabular}{|c|c|c|c|}
\hline Gene ID & Primer Name & Sequence $\left(5^{\prime}-3^{\prime}\right)$ & $\operatorname{Tm}\left({ }^{\circ} \mathrm{C}\right)$ \\
\hline \multirow{2}{*}{ Gh_A08G0457 } & FLA7-F & GTTGCAATGAGCACAAGCCCGACACCG & 65.8 \\
\hline & FLA7-R & GTACGAAAATCGTAATGCCTTCAACCG & 58.2 \\
\hline \multirow{2}{*}{ Gh_D02G0381 } & LTP-F & TTTACCACСССАTTCAAATGATACCC & 57.2 \\
\hline & LTP-R & TTAGGAGATGGTGGTCCAAGTTGAAG & 58.3 \\
\hline \multirow{2}{*}{ Gh_A11G0727 } & PME2-F & CTTCGAAGCGGTCCGGAAGTGGAAGG & 64.7 \\
\hline & PME2-R & CACTGCCTGATGGTTAGTAGGACCAG & 60.4 \\
\hline \multirow{2}{*}{ Gh_D08G1266 } & MYB46-F & CTGATATCAGAAGAGGGAGATTTACTC & 54.2 \\
\hline & MYB46-R & AGTACTTGGTGGAGGAGCAGTGCTTG & 62.3 \\
\hline \multirow{2}{*}{ Gh_D09G2166 } & CESA-G3-F & TCAACAACATTCCACGACGGGTGACG & 62.9 \\
\hline & CESA-G3-R & GTCGCAGTCAAGCATTAGAATATATGG & 55.7 \\
\hline \multirow{2}{*}{ Gh_D02G0079 } & UGT74F2-F & GATGAAGGCAGATTCTTTGAAGTTAGG & 55.9 \\
\hline & UGT74F2-R & TATGTAATTCACAGCGCACGCCTGCG & 63.3 \\
\hline \multirow{2}{*}{ Gh_D06G0375 } & 6-PGL2-F & GGAAAACGTAGTCCCTAAGCGACACC & 60.9 \\
\hline & 6-PGL2-R & GACAGTCGGTTGATCGGGAAACCTG & 61.3 \\
\hline \multirow{2}{*}{ Gh_A11G0400 } & PER72-F & CTTCCAAGACAGTTGGAGGAGGATAC & 58.4 \\
\hline & PER72-R & AGCTGAGTTCCTGTTTGGATTCGACC & 60.7 \\
\hline \multirow{2}{*}{ Gh_A08G0780 } & E3-F & GATTCCTTGTTCTCATGCCAAGATCC & 57.8 \\
\hline & E3-R & TAACTATTGGTTTTGCTCCAGGGAGC & 59.0 \\
\hline \multirow{2}{*}{ Gh_A01G0064 } & B3-F & ACAGAGGATAAGCTCTCTCAAGCAGG & 59.6 \\
\hline & B3-R & TGCTCGTTCCATTACAGCCAATTTGG & 59.9 \\
\hline \multirow{2}{*}{ Gh_A04G0106 } & ERF3-F & CTCGAATACTACCGCTTTTCCGATCG & 59.0 \\
\hline & ERF3-R & GATGATATTTCCGGTCCGACACAACC & 59.4 \\
\hline \multirow{4}{*}{ Gh_A04G0473 } & RPS5-F & GCCTCACTGCAAGACCCAACCAAGTC & 63.6 \\
\hline & RPS5-R & GCACTGAGCCTTTCTAAAACGCTTAAC & 58.4 \\
\hline & $18 \mathrm{~S}$ rRNA-F & GGAATTGACGGAAGGGCACCACCAGGC & 78.0 \\
\hline & 18S rRNA-R & GGACATCTAAGGGCATCACAGACCTG & 68.0 \\
\hline
\end{tabular}

Table S2. Association of seven chromosome 25 specific SSR markers with fiber traits.

\begin{tabular}{ccc}
\hline SSR marker & Fiber trait & References \\
\hline BNL3436 & Fineness & Mei et al. 2014 [79] \\
BNL3806 & Length, strength & Zhang et al. 2015 [80] \\
& Length & Abdurakhmonov et al. 2009 [81] \\
& Micronaire, elongation, uniformity & Sun et al. 2012 [82] \\
DPL0067 & Length & Zhang et al. 2015 [80] \\
& Elongation, micronaire & Sun et al. 2012 [82] \\
GH220 & Elongation & Liang et al. 2013 [83] \\
BNL0827 & Strength & Qin et al. 2015 [84] \\
BNL1064 & Uniformity & Sun et al. 2012 [82] \\
BNL1440 & Strength, length, uniformity, micronaire & Sun et al. 2012 [82] \\
\hline
\end{tabular}

Table S3. List of differently expressed genes (DEGs) in CS-B25 10 DPA fibers. https://gitlab.com/IGBB/OBL/ma.csb25-fiber/raw/master/Table\%20S3.xlsx 